\title{
High-order local time stepping on moving DG spectral element meshes
}

Andrew R Winters and David A Kopriva

The self-archived postprint version of this journal article is available at Linköping University Institutional Repository (DiVA):

http:/ / urn.kb.se/ resolve?urn=urn:nbn:se:liu:diva- 156870

N.B.: When citing this work, cite the original publication.

The original publication is available at www.springerlink.com:

Winters, A. R, Kopriva, D. A, (2014), High-order local time stepping on moving DG spectral element meshes, J ournal of Scientific Computing, 58(1), 176-202.

https:/ / doi.org/ 10.1007/ s10915-013-9730-z

Original publication available at:

https:/ / doi.org/ 10.1007/ s10915-013-9730-z

Copyright: Springer (part of Springer Nature) (Springer Open Choice Hybrid Journals)

http:// www.springer.com/gp/products/journals 


\title{
HIGH-ORDER LOCAL TIME STEPPING ON MOVING DG SPECTRAL ELEMENT MESHES
}

\author{
ANDREW R. WINTERS* AND DAVID A. KOPRIVA
}

\begin{abstract}
We derive and evaluate an explicit local time stepping (LTS) integration for the discontinuous Galerkin spectral element method (DGSEM) on moving meshes. The LTS procedure is derived from Adams-Bashforth multirate time integration methods. We also present speedup and memory estimates, which show that the explicit LTS integration scales well with problem size. Time-step refinement studies with static and moving meshes show that the approximations are spectrally accurate in space and have design temporal accuracy. The numerical tests validate theoretical estimates that the LTS procedure can reduce computational cost by as much as an order of magnitude for time accurate problems.
\end{abstract}

Keywords: Discontinuous Galerkin Spectral Element Method, DGSEM, Moving Mesh, Arbitrary Lagrangian-Eulerian, Multirate Time Integration, Conservation Laws

\section{INTRODUCTION}

Various applications in engineering and science can be posed as a hyperbolic system of conservation laws on domains with moving boundaries. Examples can be found in fluid dynamics $[17,31,35]$, wave scattering from moving objects in acoustics [36, 37, 38, 39], electromagnetism $[4,8,14,22,23,30]$, and biology $[2,3,15,24,25,33,34,46]$.

A common way to approximate solutions to problems with moving boundaries is to use an arbitrary Lagrangian-Eulerian (ALE) formulation [1]. In the ALE formulation, one maps a time dependent domain, $\Omega_{t}$, that has moving boundaries onto a fixed reference domain, $\Omega$. In the process, conservation law equations in the original domain are transformed to conservation law equations in the reference domain $[17,31,35]$. In the numerical approximation on the reference domain, the new set of equations depends on the mesh velocity.

To design methods for problems on a moving domain we will subdivide $\Omega_{t}$ into $K$ elements, use an ALE formulation on each element, and discretize on the reference domain with a highorder discontinuous Galerkin spectral method. The development and application of discontinuous Galerkin spectral methods for static domains are reviewed in $[6,9,27]$.

The problem with a high-order discontinuous Galerkin spectral element method (DGSEM) is that the systems are stiff. The maximum stable time step allowed by the CFL condition is dictated by the smallest elements in the mesh and the polynomial order of the approximation $[9,10]$. If refinement in the mesh is restricted to a small region, then a very small time step in the entire computational domain has a high computational cost [13]. Also, global time stepping methods are computationally inefficient on moving meshes. They must recompute the geometry of large moving elements on the time scale of the small elements. For the ALE-DGSEM the computation of an element's geometric properties is expensive $[7,27]$. If the time step needed

Department of Mathematics, The Florida State University, Tallahassee, FL 32306, USA

E-mail addresses: awinters@math.fsu.edu. 
for stability, $\Delta t_{s t a b}<\Delta t_{a c c}$, the time scale required for accuracy, the problem is stiff for an unphysical reason.

The unphysical stiffness is increased since the selection of stable time steps for the DGSEM on quadrilateral meshes with local refinement is ad hoc. Toulorge and Desmet [47] studied the effect that a triangular element's shape has on the time step of the DGSEM. They found a precise relationship between the shortest edge of a triangle and the maximum Courant number for $N \leq 10$. Similar studies are not yet available for quadrilaterals.

A natural response to the restrictive time steps required for stability by explicit global time stepping methods is to switch to implicit methods. Unfortunately, the matrices in spectral methods are dense, so the matrix solvers for implicit schemes are expensive [28]. To be effective, the increase in time step must be enough to offset the increased work per time step. For steadystate problems, when time accuracy is not an issue, implicit methods perform well [5, 28, 48]. However, for three dimensional problems or as the polynomial order $N$ increases, the memory requirements to store arrays for linear solvers and preconditioners may become prohibitive [28, 48]. For time accurate computations, where time steps are limited also by accuracy, much of the advantage of implicit methods can be lost [28].

Alternatively, explicit local time stepping (LTS) schemes have been developed to allow each element, or groups of elements, to evolve at their largest, stable time step [12, 16, 19, 21] while retaining time accuracy. For instance, Constantinescu and Sandu [11, 12] devised second order multirate explicit methods for hyperbolic conservation laws, using Runge-Kutta and AdamsBashforth schemes, respectively.

Discontinuous Galerkin methods are well-suited for the development of explicit LTS integrators because the approximation is local to each element [27]. For example, Lörcher et al. [32] derive consistent, high order LTS for discontinuous Galerkin methods that use Cauchy-Kovalevskaya time integration. The Cauchy-Kovalevskaya procedure also motivated the development of an arbitrary high-order derivatives (ADER) discontinuous Galerkin approximation for elastic wave equations [16] and the 3D Maxwell's equations [43]. Combining the idea of a multirate linear multistep method of Gear and Wells [20], Stock [41] derived many two-rate Adams-Bashforth LTS schemes. For the damped wave equation, Grote and Mitkova [21] derived continuous and discontinuous Galerkin approximations with Adams-Bashforth schemes up to order four. A second order LTS integrator that conserves the discrete energy in each element was proposed by Ezziani and Joly [18]. Krivodonova [29] developed a conservative, second order LTS strategy using Heun's method for use with non-linear conservation laws. Finally, Gassner et al. [19] created a high-order accurate predictor-corrector method for local time stepping using continuous extension Runge-Kutta (CERK).

In this paper, we design and evaluate an explicit LTS integrator for use with the DGSEM for moving or static meshes. We also derive estimates of memory requirements and CPU time savings. We follow an approach similar to that of Stock [41], except that the LTS procedure is simplified by the weak coupling arising from grid induced stiffness. We provide computations for a third order in time LTS procedure, but extension to arbitrary order in time is straightforward.

The paper is organized as follows: In Sec. 2, we present the conservation laws in curvilinear coordinates for moving domains and the semi-discrete discontinuous Galerkin spectral element approximation (DGSEM). In Sec. 3, we derive the explicit local time stepping (LTS) procedure and theoretical predictions for memory usage and cpu savings. In Sec. 4, we present four numerical examples. To validate the LTS strategy, we present a time-step refinement study on static and moving meshes in Sec. 4.1. In Sec. 4.2 we determine the actual speedup in computation time of the LTS method compared to a global time stepping method on four test meshes. We present conclusions in Sec. 5. 


\section{Moving Mesh Discontinuous Galerkin Spectral Element Method}

We start with a short review of discontinuous Galerkin spectral element methods on moving domains, whose derivation uses an explicit method of lines approach.

2.1. Discretization in Space of an ALE Conservation Law. The moving mesh DGSEM is derived from the weak form of a system of conservation laws

$$
\mathbf{q}_{t}+\nabla \cdot \mathscr{F}=0
$$

on the moving domain $\Omega_{t}$. To obtain the weak form, we multiply by an $\mathscr{L}^{2}\left(\Omega_{t}\right)$ test function $\varphi(\mathbf{x})$, and integrate in space over the moving domain

$$
\int_{\Omega_{t}}\left(\mathbf{q}_{t}+\nabla \cdot \mathscr{F}\right) \varphi d \mathbf{x} .
$$

For the spectral element method, we subdivide the physical domain $\Omega_{t}$ into $K$ non-overlapping, moving quadrilateral elements, $e_{k}$. We break the integrals over the entire domain into the sum of integrals over the moving elements

$$
\sum_{k=1}^{K}\left\{\int_{e_{k}}\left(\mathbf{q}_{t}+\nabla \cdot \mathscr{F}\right) \varphi d \mathbf{x}\right\}=0
$$

Since the test function $\varphi$ is arbitrary, each element contributes

$$
\int_{e_{k}}\left(\mathbf{q}_{t}+\nabla \cdot \mathscr{F}\right) \varphi d \mathbf{x}=0
$$

to the total integral (2.1.3).

The problem we solve is formulated as a system of conservation laws (2.1.1) where $\mathscr{F}$ is the vector flux on a time dependent domain, $e_{k}$. We create a transformation $(\mathbf{X}, t)=\mathbf{X}(\xi, \tau)$ to map the computational coordinates in the reference cube $\xi=\left(\xi^{1}, \xi^{2}, \xi^{3}\right)$ to physical coordinates $\mathbf{X}=(x, y, z) \in e_{k}[27]$. We have three covariant basis vectors, $\mathbf{a}_{i}$, computed directly from the transformation

$$
\mathbf{a}_{i}=\frac{\partial \mathbf{X}}{\partial \xi^{i}}, \quad i=1,2,3 .
$$

From the covariant basis vectors one derives the contravariant basis vectors, $\mathbf{a}^{i}$, scaled by the Jacobian of the transformation, $\mathcal{J}$,

$$
\mathcal{J} \mathbf{a}^{i}=\mathcal{J} \nabla \xi^{i}=\mathbf{a}_{j} \times \mathbf{a}_{k} \quad(i, j, k) \text { cyclic. }
$$

The conservation law in the time-dependent domain transforms to a conservation law equation in the reference domain $[17,31,35]$

$$
\tilde{\mathbf{q}}_{\tau}+\nabla_{\xi} \cdot \mathcal{F}=0
$$

where

$$
\tilde{\mathbf{q}}=\mathcal{J} \mathbf{q}, \quad \text { and } \quad \mathcal{F}=\mathcal{J} \mathbf{a}^{i} \cdot\left(\mathscr{F}-\mathbf{q} \mathbf{x}_{\tau}\right) .
$$

For complete details on the transformation of the the conservation law see Acosta and Kopriva [1]. Notice that in the transformed variables (2.1.8) the solution $\tilde{\mathbf{q}}$ incorporates the time-dependent Jacobian $\mathcal{J}$ and the flux $\mathcal{F}$ incorporates the mesh velocity $\mathbf{x}_{\tau}$.

To ensure that the discrete approximation does not introduce spurious waves, a constant solution of (2.1.7) should remain constant for all time [26]. In fluid dynamics this is often 
referred to as free-stream preservation. The conditions that guarantee free-stream preservation for the transformation $\mathbf{X}(\xi, \tau)$ are three metric identities [26]

$$
\sum_{i=1}^{3} \frac{\partial\left(\mathcal{J} a_{n}^{i}\right)}{\partial \xi^{i}}=0, \quad n=1,2,3,
$$

and the geometric conservation law (GCL) [35]

$$
\mathcal{J}_{\tau}-\sum_{i=1}^{3} \frac{\partial}{\partial \xi^{i}}\left(\mathcal{J} \mathbf{a}^{i} \cdot \mathbf{x}_{\tau}\right)=0 .
$$

The metric identities (2.1.9) state that the divergence of a constant flux vanishes in the reference cube. The geometric conservation law (2.1.10) states that for a constant solution the control volume is conserved. As we stated earlier, these four identities should be satisfied discretely at each time step to maintain free-stream preservation [1, 26, 42].

In what follows we will reduce the discussion to two dimensional problems. The ALE formulation (2.1.7), metric identities (2.1.9), and GCL (2.1.10) have been given in three dimensions since it is simpler to formulate how the equations transformed in 3D. However, we now consider the approximation of the conservation law (2.1.7) on the reference square. For a two dimensional problem note that the vector flux $\mathscr{F}=(\mathbf{f}, \mathbf{g})$ in the transformed variables $(2.1 .8)$.

Next we determine how the reference square is mapped to a moving quadrilateral element. Each element $e_{k}$ is bounded by four moving curves $\Gamma_{j}, j=1,2,3,4$. Kopriva [26] showed that the metric identities (2.1.9) are satisfied discretely if the boundaries of a quadrilateral element are approximated by a polynomial with order equal to or less than the order of the polynomial approximation of the solution. Therefore, to satisfy the metric identities (2.1.9) we use an isoparametric approximation in which each curve $\Gamma_{j}(s, \tau), s \in[-1,1]$ is approximated by a polynomial of order $N$. We write each curve in the Lagrange form

$$
\Gamma=\sum_{j=0}^{N} \Gamma\left(s_{j}, \tau\right) \ell_{j}(s) .
$$

A common transformation between the reference square and any curve-sided quadrilateral is transfinite interpolation with linear blending [27]. The mapping between the coordinates of the reference square $E$ and the physical coordinates $\mathbf{X}$ is

$$
\begin{aligned}
\mathbf{X}(\xi, \tau) & =\frac{1}{2}\left[(1-\xi) \Gamma_{4}(\eta, \tau)+(1+\xi) \Gamma_{2}(\eta, \tau)+(1-\eta) \Gamma_{1}(\xi, \tau)+(1+\eta) \Gamma_{3}(\xi, \tau)\right] \\
& -\frac{1}{4}\left[(1-\xi)\left\{(1-\eta) \Gamma_{1}(-1)+(1+\eta) \Gamma_{3}(-1)\right\}\right. \\
& \left.+(1+\xi)\left\{(1-\eta) \Gamma_{1}(1)+(1+\eta) \Gamma_{3}(1)\right\}\right] .
\end{aligned}
$$

Since the boundaries are approximated by polynomials, the transformation (2.1.12) is also a polynomial. Therefore, the calculation of the metric terms $\mathbf{X}_{\xi}$ and $\mathbf{X}_{\eta}$ is straightforward. We use the metric terms to approximate the normal vectors and time dependent Jacobian. See Kopriva [26] for more details on quadrilateral mappings for spectral methods. The mesh velocities $\mathbf{X}_{\tau}$ can also be calculated given the time derivatives of the element's boundary curves [1].

From the map $\mathbf{X}(\xi, \tau)$, (2.1.12), the weak form on each moving element (2.1.4) becomes

$$
\iint_{E}\left(\tilde{\mathbf{q}}_{\tau}+\nabla_{\xi} \cdot \mathcal{F}\right) \varphi d \xi d \eta=0 .
$$

We integrate by parts once in (2.1.13), separating boundary and interior contributions, to find

$$
\iint_{E} \tilde{\mathbf{q}}_{\tau} \varphi d \xi d \eta+\int_{\partial E} \mathcal{F} \varphi \cdot \hat{n} d S-\iint_{E} \mathcal{F} \cdot \nabla_{\xi} \varphi d \xi d \eta=0
$$


Note that $\mathcal{F}$ is the vector of contravariant fluxes $\mathcal{F}=(\tilde{\mathbf{f}}, \tilde{\mathbf{g}})$ given by

$$
\begin{aligned}
\tilde{\mathbf{f}} & =Y_{\eta}\left(\mathbf{f}-X_{\tau} \mathbf{q}\right)-X_{\eta}\left(\mathbf{g}-Y_{\tau} \mathbf{q}\right) \\
\tilde{\mathbf{g}} & =-Y_{\xi}\left(\mathbf{f}-X_{\tau} \mathbf{q}\right)+X_{\xi}\left(\mathbf{g}-Y_{\tau} \mathbf{q}\right),
\end{aligned}
$$

with the mapping $\mathbf{X}=(X, Y)$ and mesh velocity $\mathbf{X}_{\tau}=\left(X_{\tau}, Y_{\tau}\right)$. The contravariant fluxes $\tilde{\mathbf{f}}$ and $\tilde{\mathrm{g}}$ come from the definition (2.1.8).

We select the test function $\varphi$ to be the piecewise polynomial of degree $N$ in each direction on the reference element

$$
\varphi^{k}=\sum_{i=0}^{N} \sum_{j=0}^{N} \varphi_{i j}^{k} \ell_{i}(\xi) \ell_{j}(\eta)
$$

where $\left\{\ell_{n}\right\}_{n=0}^{N}$ is the Lagrange basis. We do not enforce continuity of the test function $\varphi$ at the element boundaries. We construct a nodal Galerkin approximation [27], so we replace the solution $\tilde{\mathbf{q}}$ and contravariant fluxes $\tilde{\mathbf{f}}$, $\tilde{\mathbf{g}}$ by polynomial interpolants written in Lagrange form

$$
\begin{aligned}
\tilde{\mathbf{q}}=\mathcal{J} \mathbf{q} \approx \mathcal{J} \mathbf{Q} & =\tilde{\mathbf{Q}}=\sum_{n=0}^{N} \sum_{m=0}^{N} \tilde{\mathbf{Q}}_{n m} \ell_{n}(\xi) \ell_{m}(\eta), \\
\tilde{\mathbf{f}} \approx \tilde{\mathbf{F}} & =\sum_{n=0}^{N} \sum_{m=0}^{N} \tilde{\mathbf{F}}_{n m} \ell_{n}(\xi) \ell_{m}(\eta), \\
\tilde{\mathbf{g}} & \approx \tilde{\mathbf{G}}=\sum_{n=0}^{N} \sum_{m=0}^{N} \tilde{\mathbf{G}}_{n m} \ell_{n}(\xi) \ell_{m}(\eta) .
\end{aligned}
$$

We substitute the polynomial representation of $\varphi(2.1 .16)$ and the polynomial approximations (2.1.17) into the weak form (2.1.14) to obtain

$$
\sum_{i=0}^{N} \sum_{j=0}^{N}\left\{\iint_{E} \tilde{\mathbf{Q}}_{\tau} \ell_{i} \ell_{j} d x d y+\int_{\partial E} \mathbf{F} \ell_{i} \ell_{j} \cdot \hat{n} d S-\iint_{E} \mathbf{F} \cdot \nabla_{\xi}\left(\ell_{i} \ell_{j}\right) d x d y\right\} \varphi_{i j}=0,
$$

where $\mathbf{F}=(\tilde{\mathbf{F}}, \tilde{\mathbf{G}})$. Since the values of $\varphi_{i j}$ are arbitrary and linearly independent,

$$
\iint_{E} \tilde{\mathbf{Q}}_{\tau} \ell_{i} \ell_{j} d \xi d \eta+\int_{\partial E} \mathbf{F} \ell_{i} \ell_{j} \cdot \hat{n} d S-\iint_{E} \mathbf{F} \cdot \nabla_{\xi}\left(\ell_{i} \ell_{j}\right) d \xi d \eta=0 .
$$

To complete the spatial discretization, we choose the location of the nodes in the approximation. We select the Lagrange basis that interpolates the Legendre-Gauss nodes, which are entirely interior to the reference element. We approximate the integrals in (2.1.19) with LegendreGauss quadrature, replace the flux at element boundaries with a numerical flux, to obtain the semi-discrete approximation of (2.1.7),

$$
\begin{aligned}
\frac{d \tilde{\mathbf{Q}}_{i j}}{d \tau}+ & \left\{\left[\tilde{\mathbf{F}}^{*}\left(1, \eta_{j}\right) \frac{\ell_{i}(1)}{\omega_{i}^{(\xi)}}-\tilde{\mathbf{F}}^{*}\left(-1, \eta_{j}\right) \frac{\ell_{i}(-1)}{\omega_{i}^{(\xi)}}\right]+\sum_{n=0}^{N} \tilde{\mathbf{F}}_{n j} \hat{D}_{i n}^{(\xi)}\right\} \\
& +\left\{\left[\tilde{\mathbf{G}}^{*}\left(\xi_{i}, 1\right) \frac{\ell_{j}(1)}{\omega_{j}^{(\eta)}}-\tilde{\mathbf{G}}^{*}\left(\xi_{i},-1\right) \frac{\ell_{j}(-1)}{\omega_{j}^{(\eta)}}\right]+\sum_{n=0}^{N} \tilde{\mathbf{G}}_{i n} \hat{D}_{j n}^{(\eta)}\right\} \\
& =\frac{d \tilde{\mathbf{Q}}_{i j}}{d \tau}+\mathbf{L}_{N}^{p}\left(\mathbf{Q}, \mathbf{X}, \mathbf{X}_{\tau}\right)=0,
\end{aligned}
$$

for $i, j=0, \ldots N$, where

$$
\hat{D}_{j k}=-\frac{D_{k j} \omega_{k}}{\omega_{j}}
$$


The matrix $\hat{D}_{j k}$ is the transpose of the standard polynomial derivative matrix $D_{k j}=\ell_{j}^{\prime}\left(\xi_{k}\right)$ scaled by the weights from the Legendre-Gauss quadrature. For complete details see [27].

The components of the numerical boundary fluxes, $\tilde{\mathbf{F}}^{*}$ and $\tilde{\mathbf{G}}^{*}$, are found by adding the static mesh numerical flux and the upwind value of the approximate mesh velocity and solution on the boundary

$$
\mathbf{F}_{\text {moving }}^{*}=\mathbf{F}_{\text {static }}^{*}-\left(\mathbf{X}_{\tau} \mathbf{Q}\right) \cdot \hat{n} .
$$

The details of the numerical flux formulas depend on the particular static flux [45].

The approximation should satisfy the GCL (2.1.10), which we rewrite in the form of a conservation law

$$
\frac{\partial \mathcal{J}}{\partial \tau}+\nabla_{\xi} \cdot \Psi=0
$$

where $\Psi=-\mathcal{J} \mathbf{a}^{i} \cdot \mathbf{x}_{\tau}$. We approximate $\Psi$ by a polynomial $\tilde{\Psi}=\tilde{E} \hat{\xi}+\tilde{H} \hat{\eta}$ where

$$
\begin{aligned}
& \tilde{E}_{i j}=-\left(X_{\tau} Y_{\eta}-Y_{\tau} X_{\eta}\right)_{i j}, \\
& \tilde{H}_{i j}=-\left(-X_{\tau} Y_{\xi}+Y_{\tau} X_{\xi}\right)_{i j} .
\end{aligned}
$$

Following the same steps as above, and integrating with the same spatial approximation as is used for the solution, the semi-discrete approximation of the GCL is

$$
\begin{aligned}
\frac{d \tilde{J}_{i j}}{d \tau} & +\left\{\left[\tilde{E}^{*}\left(1, \eta_{j}\right) \frac{\ell_{i}(1)}{\omega_{i}^{(\xi)}}-\tilde{E}^{*}\left(-1, \eta_{j}\right) \frac{\ell_{i}(-1)}{\omega_{i}^{(\xi)}}\right]+\sum_{n=0}^{N} \tilde{E}_{n j} \hat{D}_{i n}^{(\xi)}\right\} \\
& +\left\{\left[\tilde{H}^{*}\left(\xi_{i}, 1\right) \frac{\ell_{j}(1)}{\omega_{j}^{(\eta)}}-\tilde{H}^{*}\left(\xi_{i},-1\right) \frac{\ell_{j}(-1)}{\omega_{j}^{(\eta)}}\right]+\sum_{n=0}^{N} \tilde{H}_{i n} \hat{D}_{j n}^{(\eta)}\right\} \\
& =\frac{d \tilde{J}_{i j}}{d \tau}+\mathbf{L}_{N}^{J}(\mathbf{Q}, \mathbf{X})=0 .
\end{aligned}
$$

The quantity $\tilde{J}$ is a polynomial of degree $N$.

The GCL numerical fluxes, $\tilde{E}^{*}$ and $\tilde{H}^{*}$, are calculated from the upwind value at the boundary, that is, from the dot product of the outward normal vector $\hat{n}$ and the corresponding boundary mesh velocity calculated from the transfinite interpolation. For instance, $\tilde{H}^{*}\left(\xi_{i}, 1, \tau\right)$ is calculated as

$$
\tilde{H}^{*}\left(\xi_{i}, 1, \tau\right)=\mathbf{X}_{\tau}\left(\xi_{i}, 1, \tau\right) \cdot \hat{n} .
$$

The semi-discrete formulation of the system of conservation laws (2.1.20) and geometric conservation law (2.1.25) use the same spatial discretization. Therefore, we integrate an augmented system of ordinary differential equations

$$
\left.\frac{d \mathbf{U}}{d \tau}\right|_{i j}+\left.\mathbf{L}\left(\mathbf{Q}, \mathbf{X}, \mathbf{X}_{\tau}\right)\right|_{i j}=0
$$

where

$$
\mathbf{U}_{i j}=\left[\begin{array}{c}
\tilde{\mathbf{Q}} \\
\tilde{J}
\end{array}\right]_{i j} \quad \mathbf{L}_{i j}=\left[\begin{array}{c}
\mathbf{L}_{N}^{p} \\
\mathbf{L}_{N}^{J}
\end{array}\right]_{i j}
$$

Acosta et al. [1] proved that using $\mathbf{Q}_{i j}=\tilde{\mathbf{Q}}_{i j} / \tilde{J}_{i j}$ preserves constant solutions and therefore avoids the generation of spurious waves that are an artifact of the mesh motion. 
2.2. Computation of the Mesh Velocity. The time accuracy of the discrete solution of the augmented system of ODEs (2.1.28) is affected by errors introduced by the temporal discretization as well as errors in the mesh positions and mesh velocities [1].

Acosta et al. [1] casts methods to calculate the mesh velocities into three categories: $(i)$ exact differentiation of the mapping $\mathbf{X}(\xi, \tau)$, (ii) integration of an acceleration equation, and (iii) numerical differentiation of the mesh position via the time integrator (Inverse operator). Full time accuracy is obtained with any of these three methods.

We select a spring-mass dashpot analogy [35], which falls into category (ii), to compute mesh velocities. The spring-mass dashpot analogy replaces the edges of an element in a computational mesh by springs. The force exerted by the springs on a node by its neighboring nodes is calculated from Hooke's law. Controlling the motion of the mesh is done by modifying the stiffness, rest length and damping constants. Cross springs, connecting opposing corners, are included to help prevent the mesh from crossing itself. The dynamics of the coupled spring system is governed by Newton's second law of motion

$$
\mathbf{F}=m \mathbf{A},
$$

where $\mathbf{F}$ is the sum of the forces, $m$ is the mass, and $\mathbf{A}$ is the resulting acceleration.

We move the mesh in response to some arbitrary motion of a physical boundary with a springmass dashpot analogy. As a result, there is no analytical representation for the position or the velocity of the mesh nodes. Instead, the motion is computed as part of the solution process. We write the acceleration equation (2.2.1) as a system of first order ODEs using a change of variables. The motion of the mesh at each node is then described by

$$
\begin{aligned}
\frac{d \mathbf{X}}{d \tau} & =\mathbf{W}(\tau), \\
\frac{d \mathbf{W}}{d \tau} & =\mathbf{A}(\mathbf{X}, \mathbf{W}, \tau)=\frac{1}{m} \mathbf{F}(\mathbf{X}, \mathbf{W}, \tau),
\end{aligned}
$$

where $m$ is a mass, $\mathbf{X}$ is the position, $\mathbf{W}$ is the velocity, and $\mathbf{A}$ is the acceleration of the mesh.

We integrate the spring-mass dashpot ODE system (2.2.2) along with the augmented system of ODEs (2.1.27)

$$
\left[\begin{array}{c}
\mathbf{U} \\
\mathbf{X}_{a} \\
\mathbf{W}_{a}
\end{array}\right]_{\tau}+\left[\begin{array}{c}
\mathbf{L}\left(\mathbf{U}, \mathbf{X}_{a}, \mathbf{W}_{a}\right) \\
\mathbf{W}_{a} \\
\mathbf{A}\left(\mathbf{X}_{a}, \mathbf{W}_{a}, \tau\right)
\end{array}\right]=0
$$

where $\mathbf{X}_{a}$ is the approximate mesh position at a fixed time $\tau$, and $\mathbf{W}_{a}$ is the approximate mesh velocity. For simplicity we have omitted the $i j$ subscripts.

Acosta and Kopriva[1] showed that using the same time integrator to approximate the position and mesh velocity in this way is sufficient to preserve the temporal accuracy of an explicit global time stepping approximation of (2.2.3).

\section{Explicit Local Time Stepping on Moving Meshes}

We next derive an explicit local time stepping method to integrate (2.2.3) using AdamsBashforth linear multistep methods that exploits the minimal coupling between elements and is straightforward to implement. The motion of the mesh itself has little affect on the structure of the LTS method. The mapping $\mathbf{X}(\xi, \tau)$ automatically handles the computation of fluxes at moving boundaries. We describe the explicit local time stepping strategy for a two dimensional problem, with a straightforward extension to three dimensions. Lastly, the LTS strategy will be valid on static and moving meshes. On a static mesh we can use a standard, constant coefficient Adams-Bashforth method. However, a moving mesh LTS method will need a variable coefficient time integration technique, which we derive in Sec. 3.2. 
3.1. Derivation of a Local Time Stepping Procedure for the DGSEM. The LTS AdamsBashforth time integrator that we use is a simplification of a fastest first multirate integrator $[20,41]$. To reduce complexity, we assume $(i)$ the time scales have intermittent synchronization levels, (ii) the elements of the mesh are grouped so there are $m+1$ time scales, as opposed to each element having its own, and (iii) the time scales differ from one another by integer factors. A further simplification is allowed by the DGSEM spatial discretization itself because elements are coupled only through the boundary flux from immediate neighbors. Once an element evolves in time with its stable time step, it does not require additional information from the small time scales. Thus, the coupling between slow and fast time scales is also weak.

To describe a multirate time integration method, we consider the coupled ODE system $\dot{\mathbf{y}}=$ $F(t, \mathbf{y})$. For simplicity of the discussion, we assume there are two time scales, as in $[11,41]$, denoted as fast and slow. We can write the right hand side $F(t, \mathbf{y})$ for a general problem with strong coupling between fast and slow time scales as

$$
F(t, \mathbf{y})=\left[\begin{array}{ll}
F_{f f} & F_{f s} \\
F_{s f} & F_{s s}
\end{array}\right],
$$

and the state vector

$$
\mathbf{y}(t, \mathbf{y})=\left[\begin{array}{l}
\mathbf{y}_{f} \\
\mathbf{y}_{s}
\end{array}\right],
$$

where the subscript $f$ denotes the fast time scale and $s$ denotes the slow time scale. For (2.2.3) the analogy is that $\mathbf{y}_{s}$, the solution on large elements, can run on a large step size $\Delta t_{s}$ whereas $\mathbf{y}_{f}$, the solution on small elements, has to run with a much smaller step size $\Delta t_{f}$.

Formally, the diagonal terms $F_{f f}$ and $F_{s s}$ in (3.1.1) can be calculated immediately since the time scales match. However, to calculate cross terms (coupling) requires extrapolation for the fast to slow time scale calculation $F_{f s}$, and interpolation for the slow to fast time scale calculation $F_{s f}$. The difficulty comes from how to calculate the fast to slow time scale coupling accurately, since extrapolation is inherently unreliable. Fortunately, the weak temporal coupling between elements in the DGSEM causes $F_{f s}$ to vanish, leaving

$$
F(t, \mathbf{y})=\left[\begin{array}{cc}
F_{f f} & 0 \\
F_{s f} & F_{s s}
\end{array}\right] .
$$

The LTS method for the DGSEM needs only to construct time interpolants to compute the solution at intermediate times in the fast time scale.

We limit the number of time scales in our LTS integrator so that the construction of interpolants in time does not become so expensive as to negate speedup. Extra work is required to create interpolants between different time scales and evaluate the coupling terms. As such, we classify the elements of the mesh according to size into $m+1$ groups. The smallest elements are placed in Group $m$, the next smallest elements are placed in Group $m-1$, and so on. The largest elements are in Group 0. The LTS strategy that we derive allows each group of elements to evolve at its largest stable time step.

To group the elements, we assume that the quadrilateral mesh is generated using a quadtree with 2-refinement [40]. The quadtree method refines the mesh by subdividing elements using quadrilateral templates. Thus, each subsequent group of elements is approximately a factor of two smaller than the previous group. For example, elements in Group 1 are approximately half the size of elements in Group 0. If a quadrilateral mesh is not created using a quadtree with 2-refinement, say with Q-morph [44], it is still advantageous to group the elements, since a small number of groups significantly reduces the number of interpolants in time that must be constructed. It is always possible to look at the distribution of elements according to a measure of size, e.g., area. Once the distribution is created one can decide on the number of groups, $m$. 
The grouping strategy is inherently ad hoc depending on the size measure one uses and how one decides to divide the distribution of elements. Also, the time scales will differ by general integer factors rather than factors of two, but otherwise the time stepping algorithm can still be used.

After the elements are grouped according to size, the algorithm finds the largest stable time step for each group. First, the stable time step in Group 0 (the largest elements), called $\Delta t_{0}$, is found from a time step restriction similar to those given in $[10,19]$

$$
\Delta t_{0}=\frac{1}{\left|\lambda_{\max }\right|}\left(\frac{\Delta x}{2 N+1}\right) \text {, }
$$

where $N$ is the order of the approximation, $\Delta x$ is the average side length of elements in Group 0 , and $\lambda_{\max }$ is the maximum eigenvalue of the operator $\mathbf{L}_{N}^{p}$ in (2.1.20). The time step in the other groups is determined with

$$
\Delta t_{k}=\frac{\Delta t_{0}}{2^{k}}, \quad k=1, \ldots, m
$$

The time step selection strategy in (3.1.5) forces all of the elements to synchronize at intermediate times during the integration. We adopt the notation that the synchronization levels are

$$
t^{n}=n \Delta t_{0},
$$

as shown in Fig. 1.

$t^{n}$

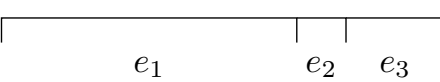

(A)

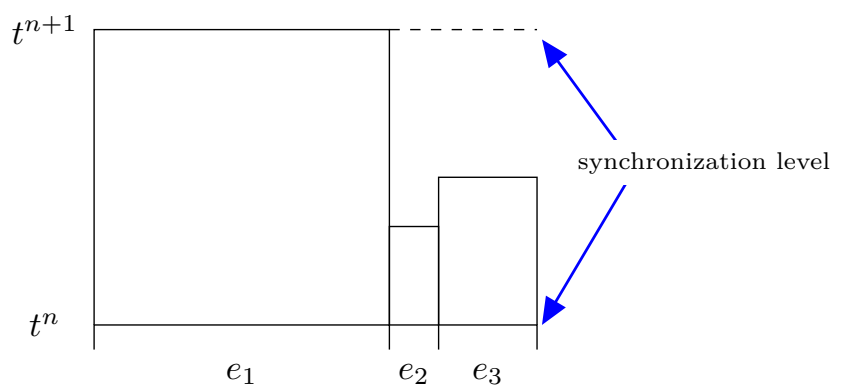

(B)

Figure 1. (A) Illustration of Synchronization LEVElS USing three Elements. (B) Each element eVolves one local time Step from a synChronization level. Note that THE LARGEST ELEMENT IS IMMEDIATELY AT THE NEXT SYNCHRONIZATION LEVEL.

The algorithm cannot integrate the system (2.2.3) at the initial synchronization level $t^{0}=0$ because Adams-Bashforth methods are not self-starting. So it uses a global time step $r+1$ order Runge-Kutta method to integrate from $t^{0}=0$ to $t^{r}=r \Delta t_{0}$. The Runge-Kutta method creates the necessary solution history on each element. We use a Runge-Kutta method of one order higher than the Adams-Bashforth method to ensure that errors in the temporal approximation are dominated by those introduced by the LTS method. Once the integration has been started by an appropriate Runge-Kutta method, the solution, GCL, and spring-mass dashpot system are at a synchronization level.

To provide a concrete discussion of the LTS method we assume that the base time integrator is a third order Adams-Bashforth method for the remainder of this section. It is straightforward to extend the LTS method to a higher temporal order if desired.

We outline the procedure to integrate the system (2.2.3) from one synchronization level, $t^{n}$, to the next, $t^{n+1}$. To augment an ALE-DGSEM implementation to include the LTS method we 
require two extra procedures: $(i)$ an evolve condition and (ii) the construction of the polynomial interpolants in time for the solution and GCL. We give detailed descriptions of these two procedures later in this section. From these descriptions we will find that the moving mesh LTS method will require a variable coefficient time integrator, which we derive in Sec. 3.2. In Sec. 3.3 , we present pseudocode for the LTS method on static or moving meshes to integrate the solution, GCL, and spring-mass dashpot system from $t^{n}$ to $t^{n+1}$.

At the synchronization level $t^{n}$ the boundary flux data necessary to evolve every element in time is available, so we sweep through the entire mesh and evolve each element one local time step as shown in Fig. 1(B). After the global time evolution from a synchronization level, the LTS method relies on an evolve condition to ensure that an element is ready to step forward in time.

One can define an evolve condition in different ways. Gassner et al. [19] use an evolve condition that checks the future time level of edge-connected neighbors to guarantee consistent edge flux calculations. However, for the LTS algorithm to operate on moving meshes using the ALE-DGSEM, an evolve condition that updates the mesh position and velocities at the smallest time scale is needed. Here, we define a local time $t^{*}$ that is intermediate between the two synchronization levels $t^{n}$ and $t^{n+1}$, that is, $t^{*} \in\left[t^{n}, t^{n+1}\right]$. Then the evolve condition is: if the local time on element $k$ is equal to $t^{*}$, then the element is ready to evolve one local time step. The algorithm also checks to see if the time on element $k$ is equal to $t^{n+1}$, since this means that the solution on element $k$ is at the next synchronization level and should not evolve. The algorithm continues to apply the evolve condition to each element until it reaches the next synchronization level, as is shown in Fig. 2(A)-2(D).

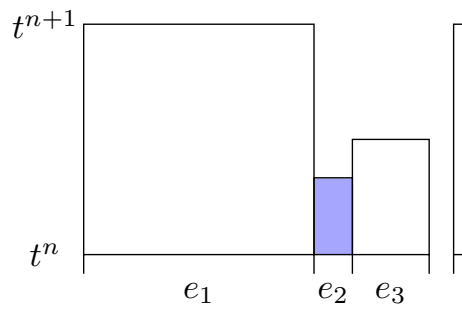

(A)

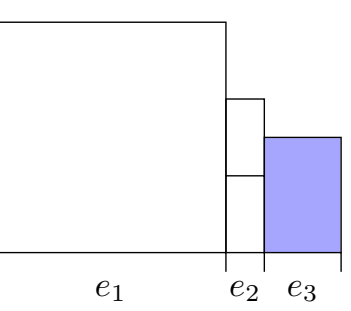

(B)

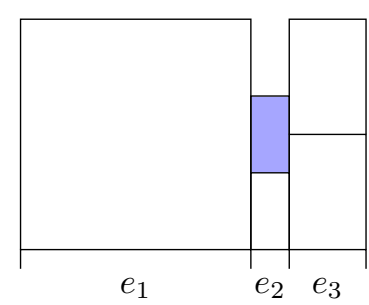

(C)

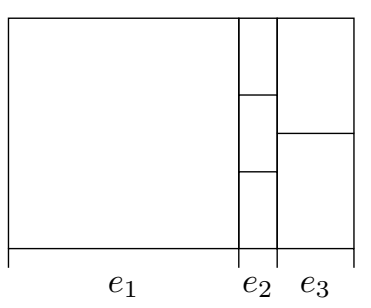

(D)

Figure 2. (A)-(C) The Progression of Repeated application of the EVOlVE CONDition to a three Element mesh. Elements Ready to eVolve are shaded. (D) EACh Element HAS REACHED THE NEXT SYNCHRONIZATION LEVEL.

The evolve condition just described guarantees that all the neighbor boundary flux data can be computed on element $k$. However, the solution on an element and the solution on the element's neighbors may be at different times, as we see in Fig. 2(A) with $e_{1}$ and $e_{2}$. We describe the procedure to calculate the boundary flux on the left interface of $e_{2}$, but the process is the same for the flux calculation at any of the element interfaces.

To compute the numerical fluxes, $\tilde{\mathbf{F}}^{*}, \tilde{\mathbf{G}}^{*}$ in $(2.1 .20)$ or $\tilde{E}^{*}, \tilde{H}^{*}$ in $(2.1 .25)$ at the intermediate time, the algorithm must construct a solution on the boundary. We show the procedure to construct the intermediate value in time in Fig. 3(A)-(C). To calculate the boundary flux at the left interface of $e_{2}$, the algorithm obtains the solution from the left using the polynomial interpolant in time. The solution from the right is found from the known value. The left and right solutions are sent to the numerical fluxes to complete the calculation.

The algorithm uses the three values of the solution history on the boundary to construct a polynomial interpolant in time as required by the third order Adams-Bashforth method time 


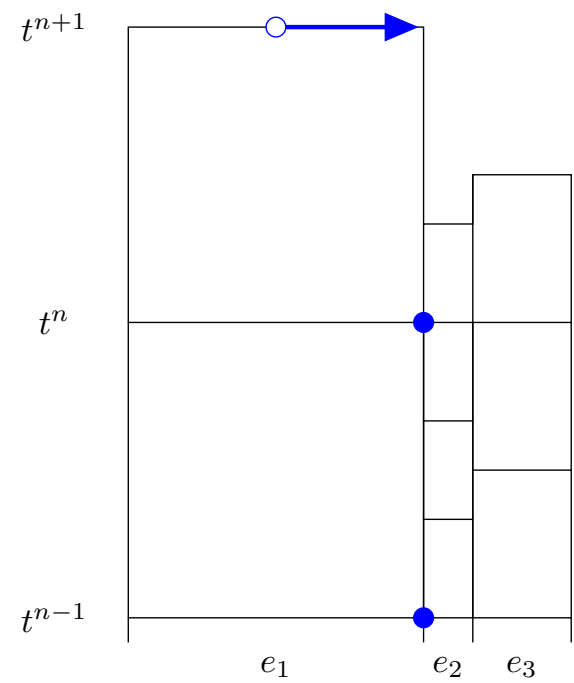

(A)

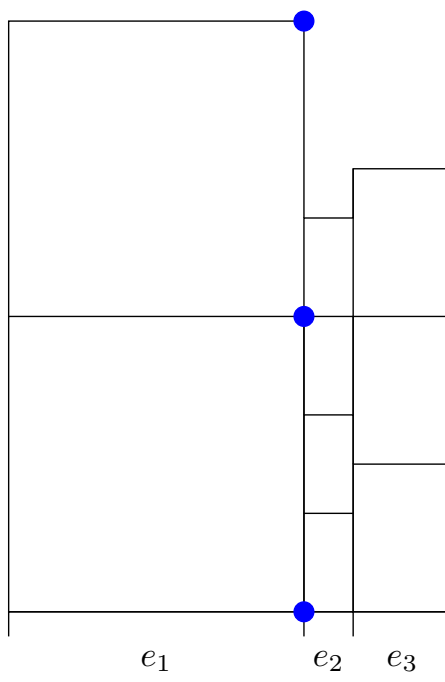

(B)

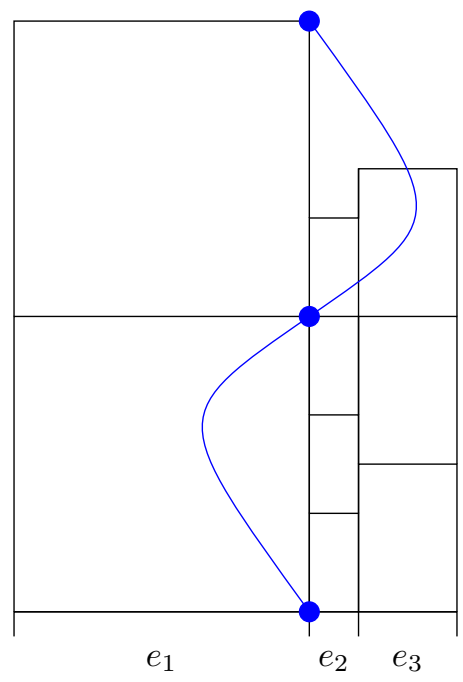

(C)

Figure 3. (a) Solution history for $e_{1}$ on the boundary and projection of the SOlution onto the Boundary at $t^{n+1}$. (B) ThreE nOdes USED to CREATE THE POLYNOMial interpolant in time. (C) Fully COnstructed polynomial interpolant in time.

integrator. The implementation stores a history of solution values on the boundary at two previous time steps, $t_{1}^{n+1}-\Delta t_{1}$ and $t_{1}^{n+1}-2 \Delta t_{1}$, where $\Delta t_{1}$ is the time step on $e_{1}$. This polynomial interpolant enables the algorithm to calculate the solution value on the boundary at any intermediate time in the interval $\left[t_{1}^{n+1}-2 \Delta t_{1}, t_{1}^{n+1}\right]$. The calculation of the boundary fluxes for the fast time evolving element $e_{2}$ in Fig. 2 occurs after the global evolution at the synchronization level. The implementation also stores a history of the Jacobian values, $\tilde{J}$, at two previous time steps on the boundaries. The algorithm uses the same process to construct a polynomial interpolant in time for Jacobian values as for the solution.

Note that if two neighboring elements are in the same group, the algorithm does not need to construct a time interpolant. All of the boundary information in the two neighbors is at the same time, so the numerical flux is calculated as with a global time stepping method. In the implementation, the algorithm checks if an element and its neighbors are at the same time to avoid the creation of unnecessary polynomial interpolants. This check reduces the computational cost of the AB3LTS time integrator.

The evolve condition and construct time interpolant procedures can be used on static or moving meshes, but when a mesh is moved it is possible for elements to stretch or shrink. In the development of the LTS algorithm we made the assumption that elements are placed into $m+1$ groups. The worst case for a moving mesh is that an element shrinks so that it should move from its current group, $j$, to the next smallest group, $j+1$. In that case, the time step on the shrinking element will no longer be small enough to maintain stability. Conversely, if an element stretches enough, then it should move from its current group to the next largest group, $j-1$. The reclassification of an element's size ensures that the element uses a stable time step as it changes shape, but prevents the algorithm from over-resolving the element in time.

If an element is reclassified from one group to another during the integration, the time derivative histories for the solution, $\tilde{\mathbf{Q}}$, and the Jacobian, $\tilde{J}$, are no longer in the correct time 
scale. The same is true of the history of the boundary values used to create time interpolants and compute boundary fluxes. The discrepancy in time scale introduces an $\mathcal{O}(\Delta t)$ error in the temporal approximation. We remedy this by introducing a variable coefficient Adams-Bashforth time integrator.

3.2. Variable Coefficient Adams-Bashforth Time Integrator. We derive a variable coefficient Adams-Bashforth method to retain full time accuracy for moving meshes where an element's size may be reclassified at any time. We use a Lagrange polynomial to determine the coefficients, which inherently includes a variable step size factor.

The variable coefficient Adams-Bashforth method is derived for the general ODE

$$
\frac{d y}{d t}=y^{\prime}=f(t, y) \text {. }
$$

For an $r^{\text {th }}$ order method the right hand side $f(t, y)$ of $(3.2 .1)$ is approximated by a Lagrange interpolating polynomial of the form

$$
p_{r}(t)=\sum_{j=0}^{r-1} \ell_{j}(t) f_{n-j}, \quad \text { where } \quad \ell_{j}(t)=\prod_{\substack{i=0 \\ i \neq j}}^{r-1} \frac{t-t_{n-i}}{t_{n-j}-t_{n-i}}
$$

To determine the general, variable coefficients we integrate (3.2.1) from $t_{n}$ to $t_{n+1}$ and obtain

$$
y_{n+1}=y_{n}+\int_{t_{n}}^{t_{n+1}} p_{r}(s) d s=y_{n}+\sum_{j=0}^{r-1} \beta_{j} f_{n-j}, \quad \text { with } \quad \beta_{j}=\int_{t_{n}}^{t_{n+1}} \ell_{j}(s) d s .
$$

For example, a third order method can be derived by expanding (3.2.2) to write the third order polynomial

$$
p_{3}(t)=\frac{\left(t-t_{n}\right)\left(t-t_{n-1}\right)}{\Delta t_{n-1}\left(\Delta t_{n}+\Delta t_{n-1}\right)} f_{n-2}-\frac{\left(t-t_{n-2}\right)\left(t-t_{n}\right)}{\Delta t_{n} \Delta t_{n-1}} f_{n-1}+\frac{\left(t-t_{n-2}\right)\left(t-t_{n-1}\right)}{\Delta t_{n}\left(\Delta t_{n}+\Delta t_{n-1}\right)} f_{n}
$$

where $\Delta t_{n}=t_{n}-t_{n-1}, \Delta t_{n-1}=t_{n-1}-t_{n-2}$, and $t_{n}-t_{n-2}=\Delta t_{n}+\Delta t_{n-1}$. We use the substitution $u=\left(t_{n+1}-s\right) / \Delta t_{n+1}$ to determine $\beta_{j}$ in (3.2.3) and find the third order variable coefficient Adams-Bashforth method

$$
y_{n+1}=y_{n}+\frac{\Delta t_{n+1}}{6}\left[r_{1} r_{0} \rho\left(2 r_{1}+3\right) f_{n-2}-r_{1} r_{0}\left(\frac{3}{\rho}+2 r_{1}\right) f_{n-1}+\left(2 \rho r_{1}^{2}+3 r_{1}(\rho+1)+6\right) f_{n}\right] \text {, }
$$

where

$$
r_{1}=\frac{\Delta t_{n+1}}{\Delta t_{n}}, \quad r_{0}=\frac{\Delta t_{n}}{\Delta t_{n-1}}, \quad \rho=\frac{r_{0}}{r_{0}+1} .
$$

For consistency when $\Delta t=\Delta t_{n+1}=\Delta t_{n}=\Delta t_{n-1}, r_{1}=r_{0}=1, \rho=1 / 2$, we recover the constant coefficient, third order Adams-Bashforth method

$$
y_{n+1}=y_{n}+\Delta t\left[\frac{5}{12} f_{n-2}-\frac{4}{3} f_{n-1}+\frac{23}{12} f_{n}\right] .
$$

The variable coefficient Adams-Bashforth method is $r^{\text {th }}$ order accurate. In general, the local truncation error for the variable coefficient Adams-Bashforth family of methods is

$$
\tau_{n+1}\left(\Delta t_{n-r+2}, \ldots, \Delta t_{n+r-2}\right)=\frac{1}{\Delta t_{n+r-2}} \int_{t_{n}}^{t_{n+1}} \frac{f^{(r)}(\xi, y(\xi))}{r !}\left(t-t_{n}\right)\left(t-t_{n-1}\right) \cdots\left(t-t_{n-r+1}\right) d t
$$


where $r$ is the order of the method and $\xi \in\left[t_{n}, t_{n+1}\right]$. For the third order method (3.2.5), $r=3$ and (3.2.8) becomes

$$
\tau_{n+1}\left(\Delta t_{n-1}, \Delta t_{n}, \Delta t_{n+1}\right)=\frac{1}{\Delta t_{n+1}} \int_{t_{n}}^{t_{n+1}} \frac{f^{(3)}(\xi, y(\xi))}{3 !}\left(t-t_{n}\right)\left(t-t_{n-1}\right)\left(t-t_{n-2}\right) d t
$$

The substitution $u=\left(t_{n+1}-t\right) / \Delta t_{n+1}$ yields the local truncation error

$$
\tau_{n+1}=\frac{y^{(4)}(\xi)}{72}\left(3 \Delta t_{n+1}^{3}+8 \Delta t_{n+1}^{2} \Delta t_{n}+4 \Delta t_{n+1}^{2} \Delta t_{n-1}+6 \Delta t_{n+1} \Delta t_{n}^{2}+6 \Delta t_{n+1} \Delta t_{n} \Delta t_{n-1}\right),
$$

where we used the fact that $y^{\prime}=f$, so $f^{(3)}=y^{(4)}$.

It remains to show that the variable coefficient explicit time integrator (3.2.5) retains third order temporal accuracy in each time scale. We show that the temporal approximation is third order in the largest possible time scale $\Delta t_{0}$. A similar process can be applied to show that it is third order in each of the others. From the time step selection strategy, (3.1.5), we define some real constants $a, b$, and $c$ such that

$$
\Delta t_{n+1}=a \Delta t_{0}, \quad \Delta t_{n}=b \Delta t_{0}, \quad \Delta t_{n-1}=c \Delta t_{0} .
$$

We note that, by design, the mesh motion will not change the shape of an element abruptly. It will never be the case that, for example, one of the largest elements will become one of the smallest elements in a single time step. A worst case is that an element shrinks from Group 0 to Group 2 in three time steps where the ratio of $a$ to $c$ is $4, a$ to $b$ is 2 , and $b$ to $c$ is 2 . In practice, we can expect the ratio between the constants $a, b$, and $c$ to be near 1 .

From the relationships (3.2.11), we see that the local truncation error in the largest time scale is

$$
\tau\left(\Delta t_{0}\right)=\mathcal{C} y^{(4)}(\xi) \Delta t_{0}^{3}, \quad \xi \in\left[t_{n}, t_{n+1}\right],
$$

where

$$
\mathcal{C}=\frac{1}{72}\left(3 c^{3}+8 a^{2} b+4 a^{2} c+6 a b^{2}+6 a b c\right) .
$$

The constant $\mathcal{C}$ is bounded, and is usually close to the constant of the traditional third order Adams-Bashforth, $3 / 8$. We verify by numerical example that the temporal accuracy in each time scale of the variable coefficient AB3LTS time integrator is third order in Sec. 4.1.2.

3.3. Outline of the LTS Algorithm. We present general pseudocode in Algorithm 1 (StepByABLTS) that outlines the integration of the solution from a synchronization level $t^{n}$ to the next synchronization level $t^{n+1}$. Important features of the LTS procedure are:

- It is independent of the set of equations to be solved, linear or non-linear. All one needs to provide are procedures to compute the physical and numerical fluxes.

- It is readily applied to three dimensional computations, where the time interpolants are along adjacent faces rather than edges.

- It can be any temporal order provided one extends the solution histories and uses an appropriate variable coefficient time integrator of the form (3.2.3).

The procedure requires as input the elements, the time for the next synchronization level $t^{n+1}$, a Boolean variable MovingMesh, the number of element groups $m$, and the time step for the group of smallest elements $\Delta t_{m}$. Initially, the integration is at a synchronization level, so we set a Boolean variable SYNCH. The algorithm visits every element in the mesh and progresses the solution and GCL if the element satisfies the evolve condition at $t^{*}$. The construction of any necessary time interpolants occurs in "Assemble time derivatives." The phrasing "Update solution $\tilde{\mathbf{Q}}_{k}$ and Jacobian $\tilde{J}_{k}$ " is purposefully vague. If the mesh is static the constant coefficient 
Adams-Bashforth is used. If the mesh is moving, a variable coefficient Adams-Bashforth like (3.2.5) replaces it. An array of Boolean variables keeps track of which elements have evolved. Finally, if an element has been evolved, the time derivative and boundary solution histories are updated.

The differences between the static and the moving mesh implementations are minimal: $(i)$ "Assemble time derivatives" includes an update of the element geometry to the current time $t^{*}$. This update includes the recalculation of a moving element's size using some measure, (ii) a variable coefficient time integrator, and (iii) the spring-mass dashpot system is integrated at the smallest time scale $\Delta t_{m}$.

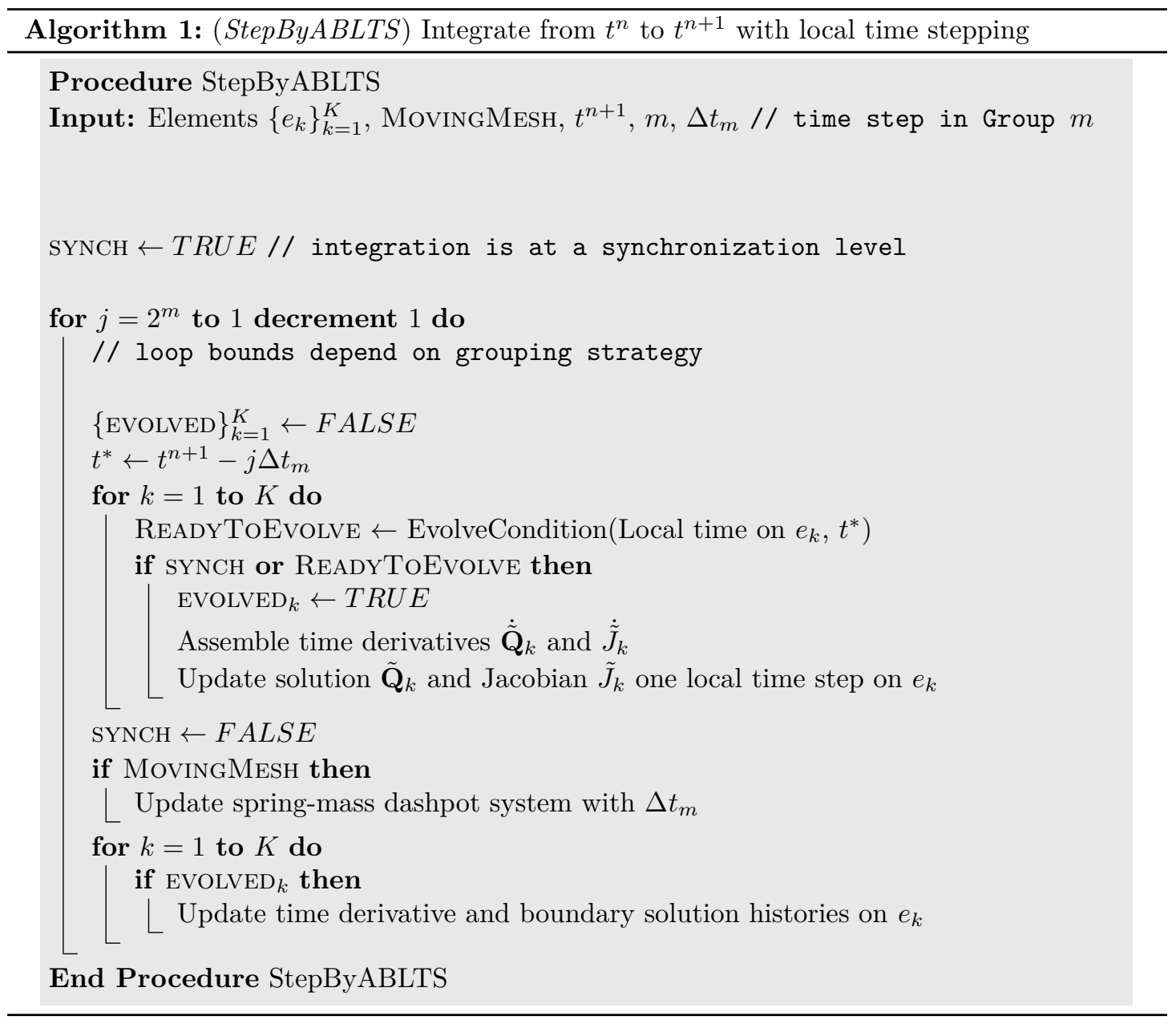

3.4. Storage and Computational Cost of the LTS Method. The ability to perform local time stepping comes at the cost of higher storage. We will compare the storage and computational cost requirements of the AB3LTS against the third order low-storage explicit Runge-Kutta (LSRK3) scheme of Williamson [49]. Thus, we compare the AB3LTS to a Runge-Kutta time integrator already optimized to be low storage. 
3.4.1. Storage Requirements of the LTS Method Compared to LSRK3. In two dimensions, the storage complexity for LSRK3 is $2(N+1)(3 N+11)$ per equation. It stores $\operatorname{six}(N+1)^{2}$ arrays and $16(N+1)$ arrays on each element for each equation to be solved. The six $(N+1)^{2}$ arrays hold the solution $\tilde{\mathbf{Q}}$, the time dependent Jacobian $\tilde{J}$, the time derivatives $\dot{\tilde{\mathbf{Q}}}, \dot{\tilde{J}}$, and an intermediate storage array $G$. In addition, each edge of the quadrilateral requires four $(N+1)$ arrays to store the solution $\tilde{\mathbf{Q}}$, the Jacobian $\tilde{J}$, and the numerical fluxes on the boundary, totaling 16 arrays.

The storage complexity for AB3LTS in two dimensions is $8(N+1)(N+5)$ per equation. It requires the time derivative at previous times, two for third order. Because elements no longer evolve at the same rate the algorithm cannot simply recalculate $\dot{\tilde{\mathbf{Q}}}$ at the previous times. The implementation therefore stores a history of the time derivatives $\dot{\tilde{\mathbf{Q}}}$ and $\dot{\tilde{J}}$ on $e_{i}$ to avoid the introduction of an $\mathcal{O}(\Delta t)$ error. The AB3LTS scheme stores eight $(N+1)^{2}$ arrays and $32(N+1)$ arrays for each equation. The eight $(N+1)^{2}$ arrays store the solution $\tilde{\mathbf{Q}}$, the time dependent Jacobian $\tilde{J}$, and the time derivatives $\dot{\mathbf{Q}}$ and $\dot{\tilde{J}}$ at the three times, $t_{i}^{n}, t_{i}^{n}-\Delta t_{i}, t_{i}^{n}-2 \Delta t_{i}$. Because the coupling is weak in the DGSEM, we do not need to store a history of the solution $\tilde{\mathbf{Q}}$ in the element. Each edge of the quadrilateral requires eight $(N+1)$ arrays that store the solution $\tilde{\mathbf{Q}}$ and Jacobain $\tilde{J}$ on the boundary at the three times, $t_{i}^{n}, t_{i}^{n}-\Delta t_{i}, t_{i}^{n}-2 \Delta t_{i}$, as well as the numerical fluxes on the boundary at time $t_{i}^{n}$, totaling 32 arrays.

The memory requirements for the local time stepping method scales well as $N$ increases. Table 1 presents the estimated factor of increase in storage for $N=4, \ldots, 10$. The storage size for these commonly used values of $N$ increases by approximately 50 percent. As $N \rightarrow \infty$, the storage of AB3LTS is 33 percent larger than LSRK3 for two dimensional problems.

The explicit local time stepping strategy remains an attractive option for three dimensional computations, again from a memory standpoint; the storage grows in $N$ identically for two dimensional or three dimensional problems. Following the same analysis as above, the storage complexity of LSRK3 and AB3LTS for a three dimensional problem is, ignoring $\mathcal{O}(1)$ terms,

$$
\begin{aligned}
& \text { LSRK3 }: 2(N+1)^{2}(3 N+11) \text { per equation, } \\
& \text { AB3LTS : } 8(N+1)^{2}(N+5) \text { per equation. }
\end{aligned}
$$

So (3.4.1) shows that the storage of AB3LTS is 33 percent larger than LSRK3 for three dimensional problems, too, as $N \rightarrow \infty$.

\begin{tabular}{lccccccc}
\hline$N$ & 4 & 5 & 6 & 7 & 8 & 9 & 10 \\
\hline Factor & 1.57 & 1.54 & 1.52 & 1.50 & 1.49 & 1.47 & 1.46 \\
\hline
\end{tabular}

TABle 1. Estimated FaCtor of InCREASE in STORAGE COMPLEXITy FOR AB3LTS versus GLOBAL TIME STEPPING LSRK3 FOR VARIOUS $N$.

3.4.2. Reducing Mesh Induced Stiffness: Theoretical Predictions. We also predict how the AB3LTS algorithm can reduce the computational work for static and moving meshes with local refinement. Again we compare the AB3LTS method with LSRK3. For each time integrator we assume that the calculation of the time derivative $\dot{\tilde{\mathbf{Q}}}$ dominates the computation. The work estimate for each time integrator uses the number of times $\dot{\tilde{\mathbf{Q}}}$ must be calculated to progress the solution from one synchronization level to the next. We include the recalculation of the element geometry as part of the assembly of the time derivative $\dot{\tilde{\mathbf{Q}}}$ to simplify the discussion of the work estimates. 
The work estimate for AB3LTS is

$$
\text { AB3LTS : } \sum_{k=0}^{m} 2^{k} \mathcal{G}(k),
$$

where $\mathcal{G}(k)$ is the number of elements in the $k^{\text {th }}$ group. Each group of elements has its own time step $\Delta t_{0}, \ldots, \Delta t_{m}$ in the AB3LTS algorithm. The LTS method stores a history of the time derivative $\dot{\tilde{\mathbf{Q}}}$ at two previous times on each element. On any element in the mesh it calculates $\dot{\tilde{\mathbf{Q}}}$ once to evolve one local time step. So, progress of the solution on the largest elements, Group 0 , from $t^{n}$ to $t^{n+1}$ requires one calculation of $\dot{\mathbf{Q}}$. From (3.1.5), we know that

$$
t^{n+1}=t^{n}+\Delta t_{0}=t^{n}+2 \Delta t_{1}=\cdots=t^{n}+2^{m} \Delta t_{m} .
$$

Thus, the elements in Group 1 require two calculations of $\dot{\tilde{\mathbf{Q}}}$ to progress the solution from $t^{n}$ to $t^{n+1}$. If we continue this argument for the number of $\dot{\tilde{\mathbf{Q}}}$ calculations in each group of elements, we find the work estimate (3.4.2).

The work estimate for LSRK3 is

$$
\text { LSRK3 : } 3 K 2^{m-1} \text {. }
$$

To derive it, we first denote the time step for the global time stepping LSRK3 to be $\Delta t_{R K}$. The region of absolute stability for third order Runge-Kutta is significantly larger than that of the third order Adams-Bashforth. Also, we take

$$
\Delta t_{R K}=\frac{\Delta t_{0}}{2^{m-1}}
$$

where $m$ is the group of the smallest elements in the mesh. The LSRK3 time integrator must calculate the time derivative $\dot{\tilde{\mathbf{Q}}}$ three times to progress the solution from $t^{n}$ to $t^{n}+\Delta t_{R K}$. From (3.4.5),

$$
t^{n+1}=t^{n}+2^{m-1} \Delta t_{R K},
$$

and we arrive at the work estimate (3.4.4).

The two time integrators perform the time derivative calculation differently. The work estimate for AB3LTS (3.4.2) does not take into account the extra computational work required by the local time stepping method to create the polynomial interpolant in time described in Sec. 3.1. We will see in the numerical verification, Sec. 4.2 , that this discrepancy can show up as an overestimate of the reduction in computation time.

To study the speedup, we generate four test meshes with a two dimensional spectral element mesh generator. The four meshes, shown in Fig. 4, are $(i)$ an inner circular boundary and refinement center, (ii) nested circles, (iii) an array of circles, and $($ iv $)$ a $[-20,20] \times[-20,20]$ domain with the NACA0012 airfoil placed at the origin. We report the predictions in Table 2. The estimates reinforce intuition: if a mesh has a high proportion of "large" elements to "small" elements then the predicted speedup is greater.

The number of elements, number of time scales, and distribution of the elements affect the predicted speedup. Histograms of the element size distributions for the four test meshes are given in Fig. 5 to help understand the predicted speedup. The "Circle Array" mesh provides an example where a couple "bad" elements introduce severe time step restrictions for global time stepping methods to remain stable. Similarly, as the mesh becomes more locally refined, the problem becomes stiffer for a global time stepping algorithm. For example, the "Circle in Circle" (Fig. 4(B)) mesh has a distribution of element sizes slightly skewed to the left (towards large elements) and a factor of 2.8 predicted speedup with its five levels of refinement. The mesh distribution of the NACA0012 airfoil (Fig. 4(D)) is also slightly skewed to the left. But it has nine levels of refinement to resolve the region around the airfoil. We predict a larger speedup on 


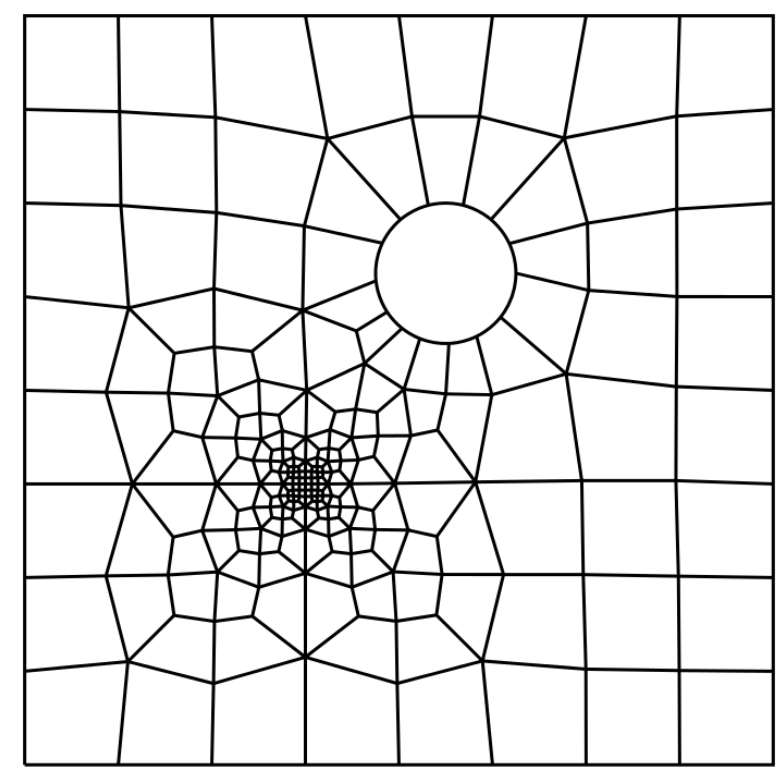

(A)

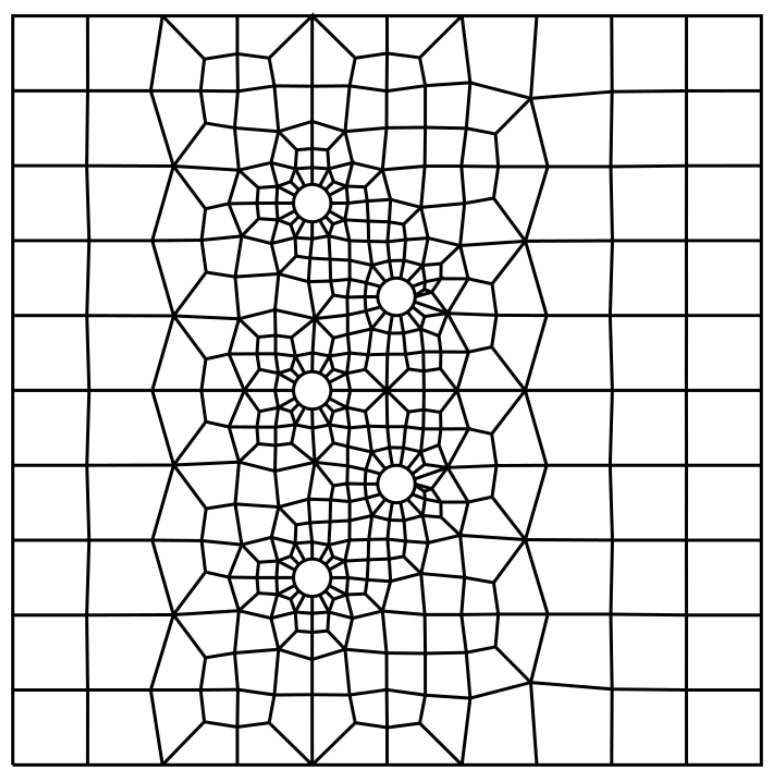

(C)

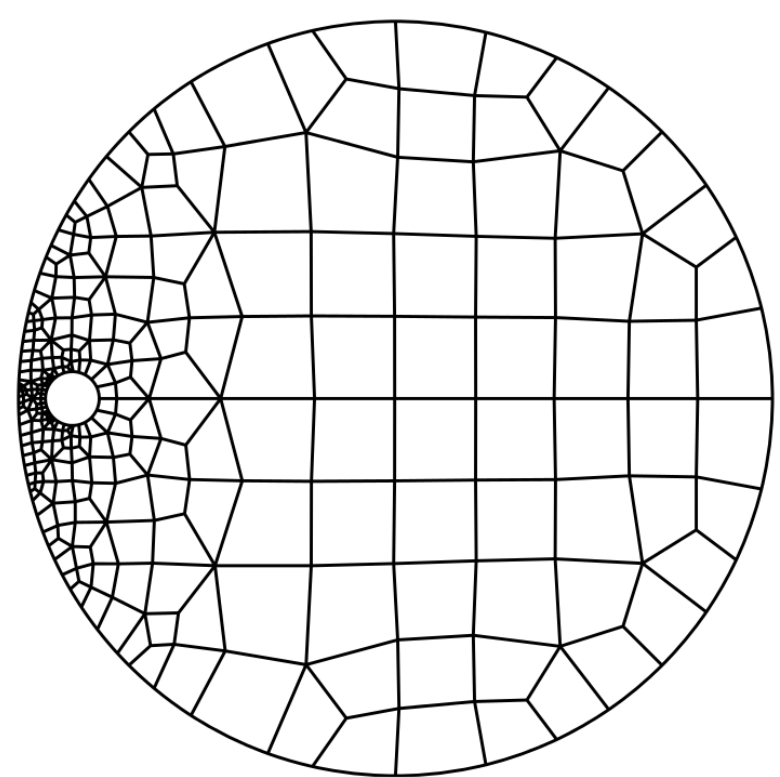

(B)

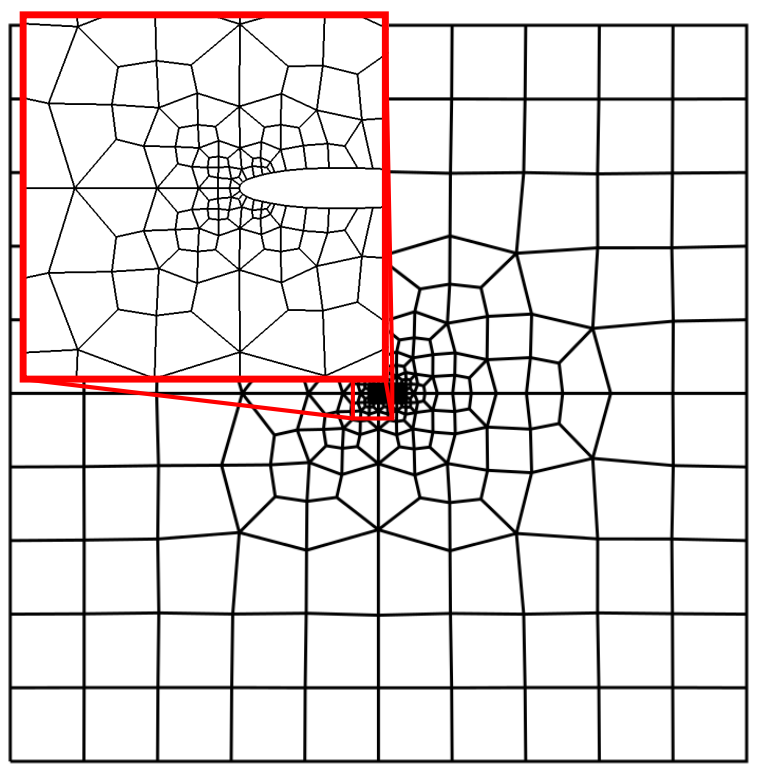

(D)

Figure 4. Several two dimensional spectral Element meshes.

the NACA0012 mesh than the "Circle in Circle" mesh when we use AB3LTS even though the two meshes have a similar distribution of element sizes.

3.4.3. For what kinds of meshes is LTS appropriate? We can use the results of the previous section to find what kinds of meshes are most appropriate for use with LTS. One would expect 


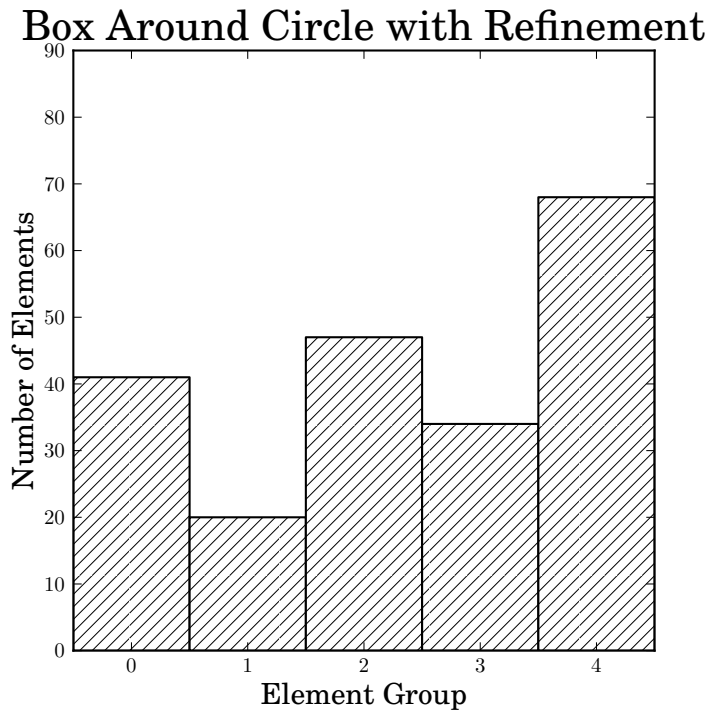

(A)

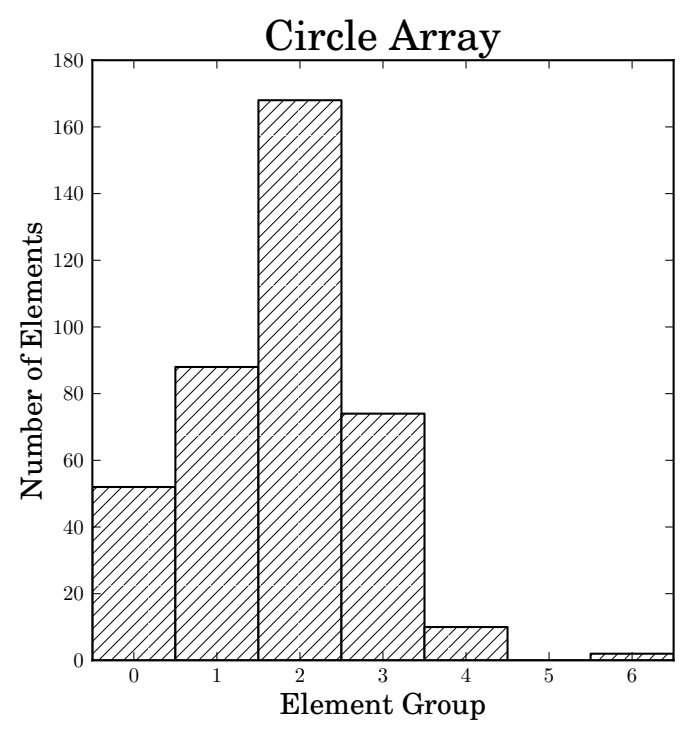

(C)

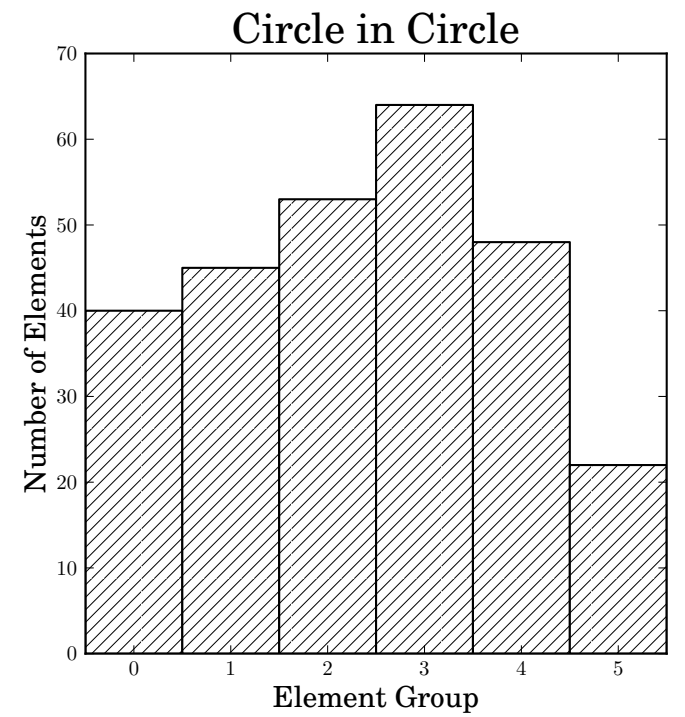

(B)

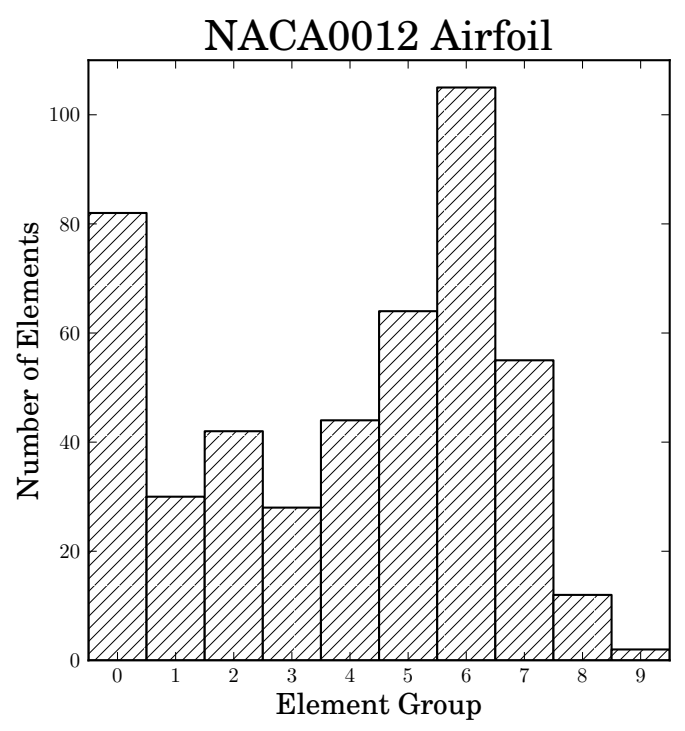

(D)

Figure 5. Histograms depict the distribution of ELEment sizes for four test meshes.

that meshes with few small elements will show the best performance, whereas for meshes with mostly small elements LTS should show little advantage. These expectations are borne out by analysis. 


\begin{tabular}{lrrr}
\hline Mesh & $\dot{\tilde{\mathbf{Q}}}$ evals LSRK3 & $\dot{\tilde{\mathbf{Q}}}$ evals AB3LTS & Predicted speedup \\
\hline Box Around Circle Refined & 2520 & 1629 & 1.6 \\
\hline Circle in Circle & 6528 & 2326 & 2.8 \\
\hline Circle Array & 18912 & 1780 & 10.6 \\
\hline NACA0012 Airfoil & 163584 & 17366 & 9.4 \\
\hline
\end{tabular}

TABLE 2. Estimations of WORK REDUCTION FOR AB3LTS versus LSRK3 ON FOUR MEShes.

The work estimates for AB3LTS (3.4.2) and LSRK3 (3.4.4) allow us to derive a theoretical speedup

$$
\mathcal{S}=\frac{1}{\frac{2}{3} \sum_{k=0}^{m} 2^{k-m} \hat{\mathcal{G}}_{k}},
$$

where $\hat{\mathcal{G}}_{k}$ is the fraction of the total number of elements in the $k^{\text {th }}$ group. For a uniform distribution of element sizes with

$$
\hat{\mathcal{G}}_{k}=\frac{1}{m+1},
$$

the speedup $\mathcal{S}(3.4 .7)$ is

$$
\mathcal{S}=(m+1) \frac{2^{m}}{2^{m+1}-1} .
$$

Asymptotically, as $m \rightarrow \infty$, i.e., the number of levels becomes large,

$$
\mathcal{S} \sim \frac{m+1}{2} .
$$

For example, nine levels of refinement should have a speedup of about five. An example of a mesh with mostly small elements is a distribution

$$
\hat{\mathcal{G}}_{k}=\frac{2^{k}}{\sum_{n=0}^{m} 2^{n}} .
$$

Its speedup would be

$$
\mathcal{S}=\frac{2^{m} \sum_{n=0}^{m} 2^{n}}{2^{2 m+1}-1} \rightarrow 1 \quad \text { as } \quad m \rightarrow \infty .
$$

If $m=9$ then $\mathcal{S}=1.006$. Thus, if the bulk of the elements are in the smallest group, the LTS procedure becomes ineffective, as one might expect. Finally, if the mesh is weighted towards large elements, e.g.,

$$
\hat{\mathcal{G}}_{k}=\frac{2^{-k}}{\sum_{n=0}^{m} 2^{-n}},
$$

we expect significant speedups. In fact,

$$
\mathcal{S}=\frac{2^{m}}{m+1} \sum_{n=0}^{m} 2^{-n}=\frac{2^{m+1}-1}{m+1},
$$

and as $m$ gets large,

$$
\mathcal{S} \sim \frac{2^{m+1}}{m+1}
$$


These examples confirm the expectation that the LTS procedure is most useful when the fraction of small elements drops off rapidly.

\section{NumERICAL EXPERIMENTS}

We provide four examples that combine the ALE-DGSEM spatial discretization with the AB3LTS time integrator. The first demonstrates spectral convergence and full time accuracy of the approximation on static and moving meshes with local refinement. The second compares the observed reduction in computational cost for the LTS procedure applied to both static and moving test meshes to the predictions in Table 2.

4.1. Convergence Study of the ALE-DGSEM with AB3LTS. We first show spectral convergence and full time accuracy in each time scale for the ALE-DGSEM with AB3LTS on static and moving meshes. For this study, we solve the wave equation written as a conservation law

$$
\left[\begin{array}{l}
p \\
u \\
v
\end{array}\right]_{t}+\left[\begin{array}{ccc}
-x_{t} & \rho c^{2} & 0 \\
1 / \rho & -x_{t} & 0 \\
0 & 0 & -x_{t}
\end{array}\right]\left[\begin{array}{l}
p \\
u \\
v
\end{array}\right]_{x}+\left[\begin{array}{ccc}
-y_{t} & 0 & \rho c^{2} \\
0 & -y_{t} & 0 \\
1 / \rho & 0 & -y_{t}
\end{array}\right]\left[\begin{array}{l}
p \\
u \\
v
\end{array}\right]_{y}=0
$$

where $\mathbf{x}_{t}=\left(x_{t}, y_{t}\right)$ is the mesh velocity. We choose initial and boundary conditions so that the solution is a Gaussian plane wave

$$
\left[\begin{array}{l}
p \\
u \\
v
\end{array}\right]=\left[\begin{array}{c}
1 \\
\frac{k_{x}}{\rho c} \\
\frac{k_{y}}{\rho c}
\end{array}\right] e^{-\frac{\left(k_{x}\left(x-x_{0}\right)^{2}+k_{y}\left(y-y_{0}\right)^{2}-c t\right)^{2}}{d^{2}}}
$$

with the wavevector $\mathbf{k}$ normalized to satisfy $k_{x}^{2}+k_{y}^{2}=1$. We take $c=1, \rho=1$, and vary $x_{0}$ and $y_{0}$ to adjust the initial position.

For the simple linear wave equation (4.1.1), the linear fluxes and exact Riemann solver are inexpensive to evaluate compared to the matrix-vector products required to compute the time derivative. Thus, numerical experiments using (4.1.1) will give worst case results for the speedup offered by the LTS method. For equations with non-linear fluxes, it will be far more expensive to compute fluxes and solve the Riemann problem relative to other operations, e.g., interpolation.

4.1.1. Convergence Study on a Static Refined Mesh. We obtain spectral accuracy in space and design accuracy in time for the DGSEM with LTS on a static mesh with local refinement. For the example, we take $d=\omega / 2 \sqrt{\ln (2)}, \omega=0.2$, and choose $x_{0}=y_{0}=-0.8$. The contour plots in Fig. 6 show the propagation of the plane wave (4.1.2) up to the final time $T=0.5$. We re-interpolate the computed solution to a uniform mesh of 10 points in each direction per element for plotting purposes.

The left of Fig. 7 shows exponential convergence in space until $N=16$, where the error is dominated by time integrator errors. Recall that we chose $\Delta t_{0}$ to be the time step on the largest elements in Group 0. Note that when the value of $\Delta t_{0}$ is halved the error in the approximation is reduced by a factor of 8 .

The right plot of Fig. 7 demonstrates design third order temporal accuracy in each of the groups of elements. Fig. 7 indicates that the error is largest on the Group 0 elements, as expected. We add a time convergence plot for each local time step level to ensure that third order convergence is achieved on every level of refinement and not just the error-dominating elements in Group 0. To produce the plot, we fixed $N=20, T=0.5$ and let $\Delta t$ range from $1 / 5000$ to $1 / 10000$. 


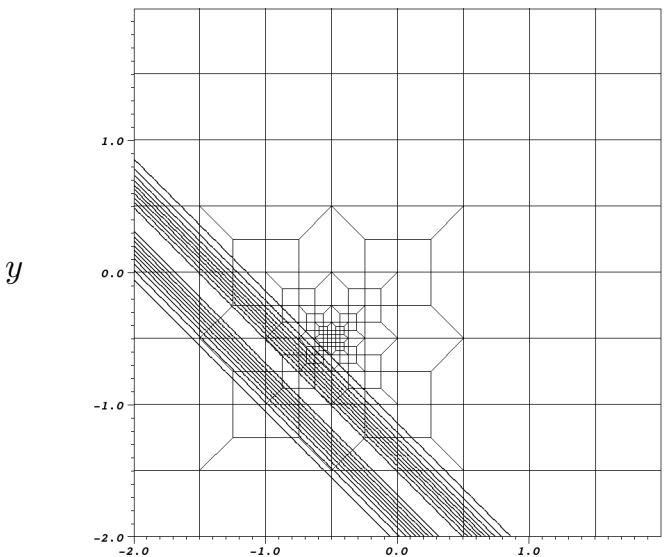

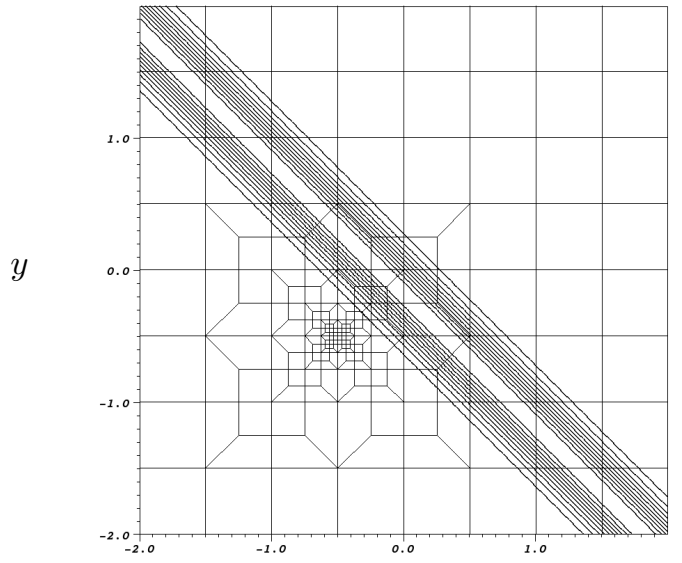

$x$

Figure 6. Contour plots of the plane wave propagation across a Refined mesh. THE OVERLAY OF SQUARES SHOWS THE ELEMENT BOUNDARIES.
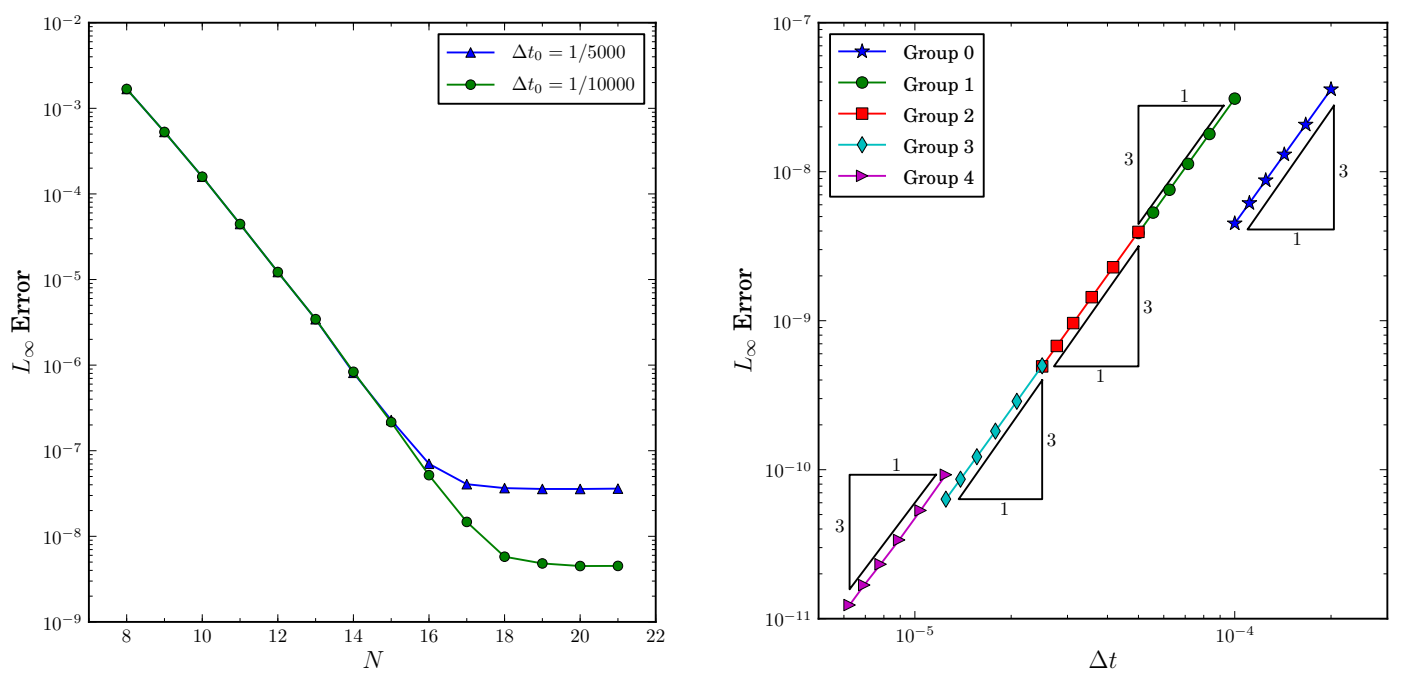

Figure 7. (left) Semi-log plot of the Spectral convergence for the DGSEM with AB3LtS ON A REFINEd MESh. (right) A LOG-LOG PLOT OF the thiRd ORDER TEMPORAL ACCURACY OF AB3LTS IN EACH GROUP OF ELEMENTS.

4.1.2. LTS on a Moving Refined Mesh. Next we demonstrate spectral convergence and design time accuracy of the AB3LTS time integrator with the ALE-DGSEM on a moving mesh with local refinement. We also verify free stream preservation. The convergence study for this numerical test exercises the variable coefficient formulation of the AB3LTS time integrator derived in Sec. 3.2 . 
Free-Stream Preservation: To show that the LTS method does not introduce spurious waves and preserves a free-stream when a mesh moves, we consider a uniform solution in time

$$
\mathbf{q}=\left[\begin{array}{l}
p \\
u \\
v
\end{array}\right]=\left[\begin{array}{l}
\pi \\
\pi \\
\pi
\end{array}\right]
$$

on a mesh with an inner circular boundary and local refinement center shown previously in Fig. 4(A). The mesh has fixed outer boundaries, whereas the inner circular boundary moves according to

$$
\begin{aligned}
& X(t)=X(0)+a \sin (f t), \\
& Y(t)=Y(0)+a \cos (f t),
\end{aligned}
$$

where $a=0.15$ and $f=\pi / 2$.

In the test of free stream preservation, we used the variable coefficient AB3LTS time integrator and a spring-dashpot model to calculate the mesh velocity. The error was calculated using the maximum norm over the component $p$ and the exact constant solution at $T=2.0$, which corresponds to a complete period in the oscillation of the inner circular boundary. Table 3 shows the computed error for double precision computations.

\begin{tabular}{ccccccc}
\hline$N$ & 4 & 6 & 8 & 10 & 12 & 14 \\
\hline Error & $2.0 \times 10^{-14}$ & $2.1 \times 10^{-14}$ & $2.1 \times 10^{-14}$ & $3.6 \times 10^{-14}$ & $6.6 \times 10^{-14}$ & $6.9 \times 10^{-14}$ \\
\hline
\end{tabular}

TABle 3. COMPUted ERror OF A CONSTANT SOlution TO THE WAVE EQUATION ON A MOVING MESH WITH LOCAL TIME STEPPING.

Convergence in Space and Time: The LTS algorithm is spectrally convergent in space and third order convergent in time for each group of elements the moving mesh. For the test problem, we choose the same parameters for the plane wave as in Sec. 4.1.1, except $x_{0}=0.0$ and $y_{0}=-0.5$. The contour plots in Fig. 8 show the propagation of the plane wave (4.1.2) up to the time $T=1.0$.

The left of Fig. 9 shows spectral convergence in the spatial error at $T=1.0$ until $N=13$, after which the error is dominated by errors introduced by the time integrator. When the value of $\Delta t_{0}$ is halved the error in the approximation is reduced by the expected factor of 8 . The convergence rate in time is also third order for each group of elements, which we show at the right in Fig. 9. Again, the error is largest on the elements in Group 0. To produce the plot we fix $N=16, T=1.0$ and let $\Delta t$ range from $1 / 3500$ to $1 / 7000$.

4.2. Reducing Non-Physical Stiffness: Numerical Verification. We now use the LSRK3 and AB3LTS to compare the actual speedup to the predicted ones found in Sec. 3.4 .2 for the same problem as before, (4.1.1) and (4.1.2). We investigate the stiffness introduced by a mesh with local refinement and its affect on the overall computation time.

We set exact boundary conditions at the edges of the computational domain and any interior boundaries, such as at the surface of the NACA0012 airfoil, like in Fig. 8. With the exact boundary conditions, any internal objects act as transparent boundaries. As such, the test problems are unphysical. However, the point is that the LTS procedure is designed to reduce the stiffness introduced by unphysical local refinement. The test problems are designed to show that it does.

We present in Table 4 the measured speedup for the static meshes listed in Table 2. We choose the time step for AB3LTS with the procedure described in (3.1.5). The time step for 
LSRK3 is given by (3.4.5). For the tests on static and moving meshes we take $N=M=6$ and $\Delta t_{0}=1 / 1000$. We see that the speedup formula (3.4.7) tends to overestimate the predicted reduction of computational cost on static meshes. This is because we neglected the additional computation time required to create the polynomial interpolant.

To examine the computational work required to construct time interpolants, we profiled a simulation with LTS on the Circle Array mesh. We find the assembly of the time derivative is $55 \%$ of the computation time. The construction of the time interpolants accounts for $4 \%$. Table 4 shows that as the number of time scales increases, the time interpolants become less important to the total computation time, and the work estimate becomes more accurate.

\begin{tabular}{lrrrrr}
\hline Mesh & LSRK3 $(\mathrm{m})$ & AB3LTS $(\mathrm{m})$ & Predicted & Measured & $\%$ Diff \\
\hline Box Around Circle & 1.03 & 0.77 & 1.6 & 1.3 & -19 \\
\hline Circle in Circle & 3.01 & 1.19 & 2.8 & 2.5 & -12 \\
\hline Circle Array & 11.50 & 1.18 & 10.6 & 9.7 & -9 \\
\hline NACA0012 Airfoil & 104.74 & 11.22 & 9.4 & 9.3 & -1 \\
\hline
\end{tabular}

TABle 4. Comparison of the predicted and measured speedup of AB3LTS versus THE GLOBAL TIME STEPPING LSRK3 ON SEVERAL STATIC MESHES.

The work estimate is better for moving meshes with local refinement. In Table 5 we show the speedup for the same test meshes used in Table 4, but now the interior boundaries move sinusoidally by (4.1.4). Recall that the work estimate included the recalculation of the element geometry in the assembly of the time derivative. The work estimate for AB3LTS (3.4.2) underestimates the benefit of LTS when the number of time scales is $\leq 5$ and can overestimate the benefit when the number of time scales is greater than 5 .

To explain the underestimation of speedup shown in Table 5, we profile the "Box Around Circle Refined" test case. We find that 38\% percent of the computation time is spent assembling the time derivative and $41 \%$ is spent updating the mesh geometry. However, the computation of the time derivative is essentially matrix-vector products, which are very efficiently computed $[27,28]$. A profile of the global time stepping Runge-Kutta method reveals that $26 \%$ of computation time is spent evaluating the time derivative and $63 \%$ is spent updating the mesh geometry. The update to the mesh geometry is always expensive regardless of time integrator, so it is advantageous to limit the number of times the procedure updates the mesh. Thus, we account for the underestimation in speedup because AB3LTS is not dominated as much by the mesh update as in LSRK3.

We explore why the work estimate becomes more accurate for meshes with a wide distribution of element sizes by profiling the "Circle Array" test case. We find that the update of the geometry of a moving element is $83 \%$ of the computation time, whereas the evaluation of the time derivatives is $7 \%$. Also, the extra computational effort to create time interpolants for the LTS procedure becomes negligible on moving meshes, just one percent of computation time. For the global time stepping LSRK3, the profile shows that $84 \%$ of computation time is spent updating the mesh geometry whereas only $7 \%$ is spent assembling the time derivative. Thus, we account for the accuracy of the work estimate in the last two entries of Table 5.

\section{Conclusion}

We have presented an Adams-Bashforth local time stepping DGSEM method for reducing mesh induced stiffness on moving and static meshes. We performed a numerical study on a 


\begin{tabular}{lrrrrr}
\hline Mesh & LSRK3 $(\mathrm{m})$ & AB3LTS $(\mathrm{m})$ & Predicted & Measured & \% Diff \\
\hline Box Around Circle & 6.69 & 1.70 & 1.6 & 3.9 & 59 \\
\hline Circle in Circle & 28.42 & 6.79 & 2.8 & 4.2 & 33 \\
\hline Circle Array & 56.00 & 5.32 & 10.6 & 10.5 & -1 \\
\hline NACA0012 Airfoil & 233.57 & 24.92 & 9.4 & 9.4 & 0 \\
\hline
\end{tabular}

TABle 5. COMParison of the PREDicted and measured speedup of AB3LTS versus THE GLOBAL TIME STEPPING LSRK3 ON SEVERAL MOVING MESHES.

static mesh to verify the spectral convergence in space and design time accuracy in each group of elements. We derived a variable coefficient time integrator for integration when the mesh moves. A numerical example on a moving mesh again verified the spectral convergence in space and design time accuracy using the new time integrator. The variable coefficient time integrator allows one to reclassify the distribution of element sizes as the mesh moves with no loss in temporal accuracy.

We showed that the memory requirements of the LTS method scales well with $N$. We also note that the DGSEM is embarrassingly parallel: the solution is calculated locally, and data is communicated only between adjacent elements. An approximation that uses the ABLTS integrator remains highly localized. Thus, ABLTS may be an attractive option for parallel computations on meshes with local refinement, modulo load balancing issues.

Numerical experiments show that time-accurate computations on meshes with a wide distribution of element sizes were up to ten times faster. The speedup of the LTS method increases as the amount of local refinement in a mesh is increased. The benefit of LTS on moving meshes is more significant because it reduces the number of updates to an element's geometry. We used a linear test problem and gave worst case results for the speedup produced by the LTS method.

Acknowledgments: This work and the authors were supported in part by the NSF grant DMS-0810925.

\section{REFERENCES}

[1] C. A. Acosta Minoli And D. A. Kopriva, Discontinuous Galerkin spectral element approximations on moving meshes, Journal of Computational Physics, 230 (2010), pp. 1876-1902.

[2] Y. Akay, M. Akay, W. Welkowitz, J. Semmlow, and J. Kostis, Noninvasive acoustical detection of coronary artery disease: a comparative study of signal processing methods, IEEE Transactions on Biomedical Engineering, 40 (1993), pp. 571-578.

[3] A. Al-Jumaily And A. Alizad, eds., Biomedical Applications of Vibration and Acoustics in Therapy, Bioeffect and Modeling, ASME Press, 2008, pp. 215-244.

[4] A. Ascoli, C. Bernasconi, And G. Cavalleri, Refraction and reflection of a nonrelativistic wave when the interface and the media are moving, Physical Review E, 54 (1996), pp. 6291-6296.

[5] P. Birken, G. Gassner, M. HaAs, and C. Munz, Efficient time integration for discontinuous Galerkin method for the unsteady $3 D$ Navier-Stokes equations, in European Congress on Computational Methods and Applied Sciences and Engineering (ECCOMAS 2012), 2012.

[6] C. Canuto, M. Hussaini, A. Quarteroni, and T. Zang, Spectral Methods: Fundamentals in Single Domains, Springer, Berlin, 2006.

[7] - Spectral Methods: Evolution to Complex Geometries and Applications to Fluid Dynamics, Springer, Berlin, 2007.

[8] D. Censor, Non-relativistic scattering by time-varying bodies and media, Progress In Electromagnetics Research, 48 (2004), pp. 249-278. 
[9] B. Cockburn And C. W. Shu, The Runge-Kutta local projection $P^{1}$-discontinuous Galerkin method for scalar conservation laws, Modélisation Mathématique et Analyse Numérique, 25 (1991), pp. 337-361.

[10] — Runge-Kutta discontinuous Galerkin methods for convection-dominated problems, Journal of Scientific Computing, 16 (2001), pp. 173-261.

[11] E. Constantinescu And A. SAndu, Multirate timestepping methods for hyperbolic conservation laws, Journal of Scientific Computing, 33 (2007), pp. 239-278.

[12] - Multirate explicit adams methods for time integration of conservation laws, Journal of Scientific Computing, 38 (2009), pp. 229-249.

[13] C. DAWSON AND R. KIRBY, High resolution schemes for conservation laws with locally varying time steps, SIAM Journal of Scientific Computing, 22 (2000), pp. 2256-2281.

[14] D. De ZutTeR, Reflections from linearly vibrating objects: plane mirror at oblique incidence, Antennas and Propagation, IEEE Transactions on, 30 (1982), pp. 898-903.

[15] J. Dennie, J. B. Mandeville, J. L. Boxerman, S. D. Packard, B. R. Rosen, and R. M. Weisskoff, NMR imaging of changes in vascular morphology due to tumor angiogenesis, Magnetic Resonance in Medicine, 40 (1998), pp. 793-799.

[16] M. Dumbser, M. KÄSER, AND E. F. TORO, An arbitrary high-order discontinuous Galerkin method for elastic waves on unstructured meshes-V. local time stepping and p-adaptivity, Geophysical Journal International, 171 (2007), pp. 695-717

[17] S. Étienne, A. Garon, And D. Pelletier, Perspective on the geometric conservation law and finite element methods for ALE simulations of incompressible flow, Journal of Computational Physics, 228 (2009), pp. 23132333.

[18] A. EzZIAni AND P. Joly, Local time stepping and discontinuous Galerkin methods for symmetric first order hyperbolic systems, Journal of Computational and Applied Mathematics, 234 (2010), pp. 1886-1895.

[19] G. Gassner, F. Hindenlang, And C. Munz, A Runge-Kutta based discontinuous Galerkin method with time accurate local time stepping, Adaptive High-Order Methods in Computational Fluid Dynamics, 2 (2011), pp. 95-118.

[20] C. W. Gear AND D. R. Wells, Multirate linear multistep methods, BIT, 24 (1984), pp. 484-502.

[21] M. Grote And T. Mitkova, High-order explicit local time-stepping methods for damped wave equations, Journal of Computational and Applied Mathematics, 239 (2013), pp. 270-289.

[22] F. Harfoush, A. Taflove, and G. Kriegsmann, A numerical technique for analyzing electromagnetic wave scattering from moving surfaces in one and two dimensions, Antennas and Propagation, IEEE Transactions on, 37 (1989), pp. 55-63.

[23] M. Ho, Scattering of electromagnetic waves from vibrating perfect surfaces: Simulation using relativistic boundary conditions, Journal of Electromagnetic Waves and Applications, 20 (2006), pp. 425-433.

[24] F. Jing, W. Xuemin, W. Mingshi, And L. Wei, Noninvasive acoustical analysis system of coronary heart disease, in Sixteenth Southern Biomedical Engineering Conference, 1997, pp. 239-241.

[25] M. Kompis, H. Pasterkamp, And G. R. WodickA, Acoustic imaging of the human chest, CHEST: American College of Chest Physicians, 120 (2001), pp. 1309-1321.

[26] D. A. Kopriva, Metric identities and the discontinuous spectral element method on curvilinear meshes, Journal of Scientific Computing, 26 (2006), pp. 301-327.

[27] _ Implementing Spectral Methods for Partial Differential Equations, Springer, 2009.

[28] D. A. Kopriva And E. Jimenez, An assessment of the efficiency of nodal discontinuous Galerkin spectral element methods, in Recent Developments in the Numerics of Nonlinear Hyperbolic Conservation Laws, R. Ansorge, H. Bijl, A. Meister, and T. Sonar, eds., vol. 120 of Notes on Numerical Fluid Mechanics and Multidisciplinary Design, Springer Berlin Heidelberg, 2013, pp. 223-235.

[29] L. Krivodonova, An efficient local time-stepping scheme for solution of nonlinear conservation laws, Journal of Computational Physics, 229 (2010), pp. 8537-8551.

[30] K. Kunz, Plane electromagnetic waves in moving media and reflections from moving interfaces, Journal of Applied Physics, 51 (1980), pp. 873-884.

[31] I. Lomtev, R. M. Kirby, And G. E. Karniadakis, A discontinuous Galerkin ALE method for compressible viscous flows in moving domains, Journal of Computational Physics, 155 (1999), pp. 128-159.

[32] F. Lörcher, G. Gassner, And C.-D. Munz, A discontinuous Galerkin scheme based on a spacetime expansion. I. inviscid compressible flow in one space dimension, Journal of Scientific Computing, 32 (2007), pp. $175-199$.

[33] G. Luo, J. J. Kaufman, A. Chinbrera, B. Bianco, J. H. Kinney, D. Haupt, J. T. Ryaby, and R. S. Siffert, Computational methods for ultrasonic bone assessment, Ultrasound in Medicine \& Biology, 25 (1999), pp. 823-830.

[34] H. Mansy, T. Royston, R. Balk, And R. SAndler, Pneumothorax detection using pulmonary acoustic transmission measurements, Medical and Biological Engineering and Computing, 40 (2002), pp. 520-525. 
[35] D. J. MAVRIPlis AND Z. YANG, Construction of the discrete geometric conservation law for high-order timeaccurate simulations on dynamic meshes, Journal of Computational Physics, 213 (2006), pp. 557-573.

[36] P. Morse And K. Ingard, Theoretical acoustics, Princeton University Press, 1987.

[37] N. Mujica, R. Wunenburger, And S. Fauve, Scattering of a sound wave by a vibrating surface, The European Physical Journal B-Condensed Matter and Complex Systems, 33 (2003), pp. 209-213.

[38] V. Ostashev, Acoustics in moving inhomogeneous media, Taylor \& Francis, 1998.

[39] J. C. Piquette, A. L. Van Buren, And P. H. Rogers, Censor's acoustical Doppler effect analysis-is it a valid method?, Journal of the Acoustical Society of America, 83 (1988), pp. 1681-1682.

[40] R. Schneiders, Refining quadrilateral and hexahedral meshes, in 5th International Conference on Grid Generation in Computational Field Simulations, 1996, pp. 679-688.

[41] A. Sтоск, Development and application of a multirate multistep AB method to a discontinuous Galerkin method based particle in cell scheme, diploma thesis, Institut für Aerodynamik und Gasdynamik Universität Stuttgart and Brown University, 2009.

[42] C. K. W. TAM, Computational aeroacoustics: an overview of computational challenges and applications, International Journal of Computational Fluid Dynamics, 18 (2004), pp. 547-567.

[43] A. Taube, M. Dumbser, C.-D. Munz, And R. Schneider, A high-order discontinuous Galerkin method with time-accurate local time stepping for the Maxwell equations, International Journal of Numerical Modelling: Electronic Networks, Devices and Fields, 22 (2009), pp. 77-103.

[44] B. H. V. Topping, J. Muylle, P. Iványi, R. Putanowicz, and B. Cheng, Finite Element Mesh Generation, Saxe-Coburg Publications, 2004.

[45] E. F. Toro, Riemann Solvers and Numerical Methods for Fluid Dynamics: A Practical Introduction, Springer, 2009

[46] A. Torres-Jimenez, S. Charleston-Villalobos, R. Gonzalez-Camarena, G. Chi-Lem, And T. AljamaCorrales, Respiratory acoustic thoracic imaging (RATHI): Assessing intrasubject variability, in Engineering in Medicine and Biology Society, 2008. EMBS 2008. 30th Annual International Conference of the IEEE, 2008, pp. 4793-4796.

[47] T. Toulorge And W. Desmet, CFL Conditions for Runge-Kutta discontinuous Galerkin methods on triangular grids, Journal of Computational Physics, 230 (2011), pp. 4657-4678.

[48] Z. WANG, High-order methods for the Euler and Navier-Stokes equations on unstructured grids, Progress in Aerospace Sciences, 43 (2007), pp. 1 - 41.

[49] J. H. Williamson, Low-storage Runge-Kutta schemes, Journal of Computational Physics, 35 (1980), pp. 4856. 


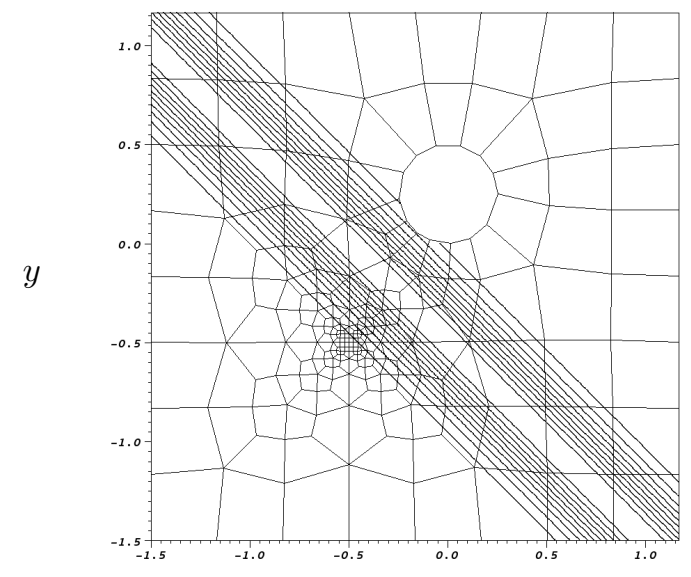

$x$

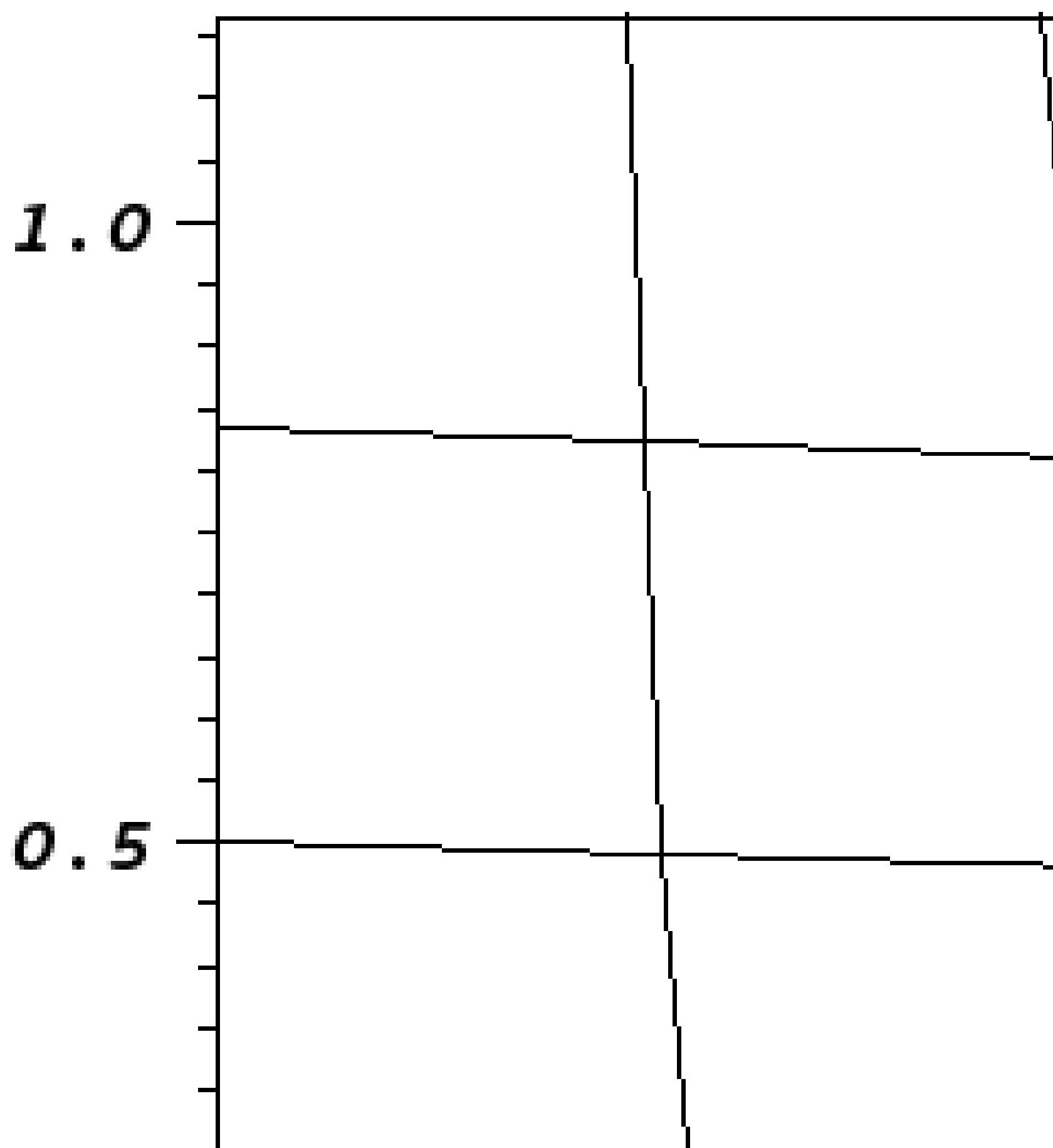



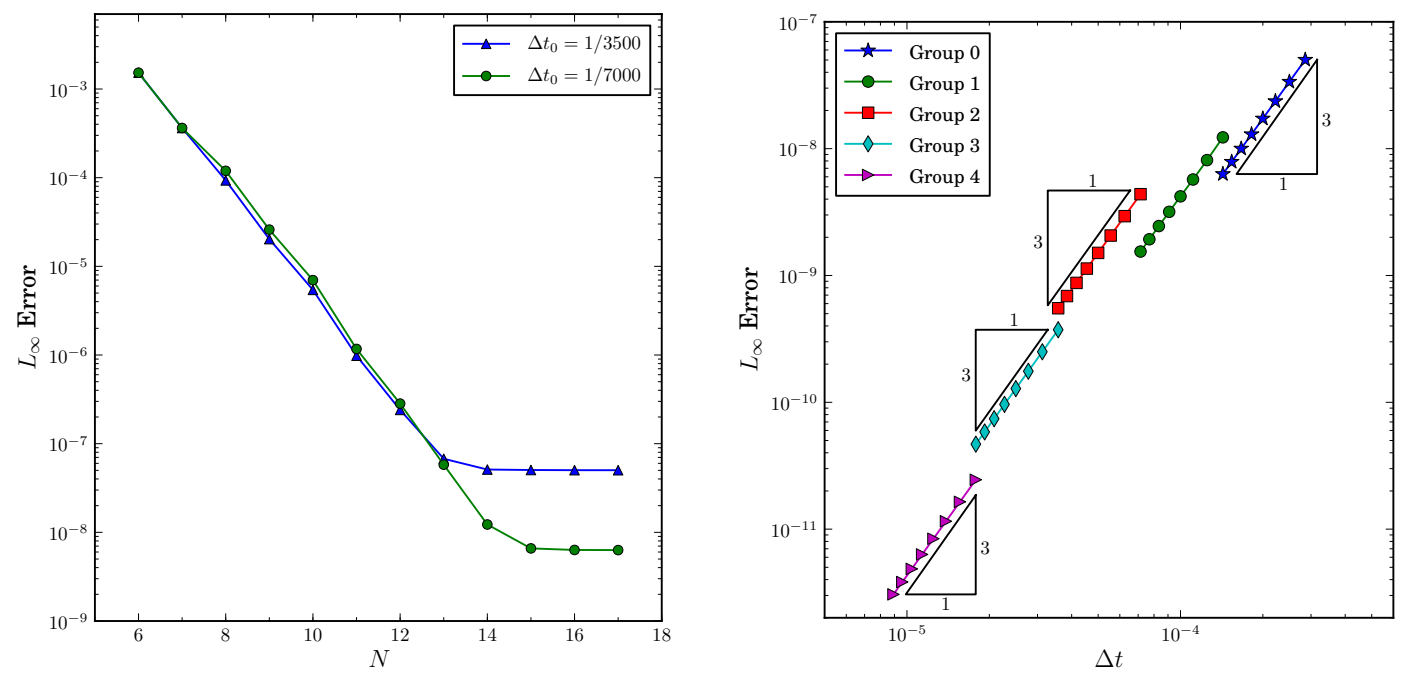

Figure 9. (left) Semi-log plot shows spectral convergence for the ALE-DGSEM with AB3LTS. (right) A LOG-LOG PLOT SHOWING THIRD ORDER TEMPORAL ACCURACY OF AB3LTS IN EACH GROUP OF ELEMENTS. 